\title{
Decompositions of locally compact contraction groups, series and extensions
}

\author{
Helge Glöckner and George A. Willis
}

\begin{abstract}
A locally compact contraction group is a pair $(G, \alpha)$, where $G$ is a locally compact group and $\alpha: G \rightarrow G$ an automorphism such that $\alpha^{n}(x) \rightarrow e$ pointwise as $n \rightarrow \infty$. We show that every surjective, continuous, equivariant homomorphism between locally compact contraction groups admits an equivariant continuous global section. As a consequence, extensions of locally compact contraction groups with abelian kernel can be described by continuous equivariant cohomology. For each prime number $p$, we use 2-cocycles to construct uncountably many pairwise non-isomorphic totally disconnected, locally compact contraction groups $(G, \alpha)$ which are central extensions

$$
\{0\} \rightarrow \mathbb{F}_{p}((t)) \rightarrow G \rightarrow \mathbb{F}_{p}((t)) \rightarrow\{0\}
$$

of the additive group of the field of formal Laurent series over $\mathbb{F}_{p}=$ $\mathbb{Z} / p \mathbb{Z}$ by itself. By contrast, there are only countably many locally compact contraction groups (up to isomorphism) which are torsion groups and abelian, as follows from a classification of the abelian locally compact contraction groups.
\end{abstract}

Classification: Primary 22D05; secondary 20E22, 20E36, 20F18, 20J06

Key words: contraction group; torsion group; extension; cocycle; section; equivariant cohomology; abelian group; nilpotent group; isomorphism types

\section{Introduction and statement of results}

An automorphism $\alpha: G \rightarrow G$ of a locally compact group $G$ is called contractive if $\lim _{n \rightarrow \infty} \alpha^{n}(x)=e$ for each $x \in G$. The article is devoted to new aspects of the theory of such locally compact contraction groups $(G, \alpha)$. Let $G_{e}$ be the connected component of the identity element $e$ of $G$. Siebert [15, Proposition 4.2] showed that $G$ has an $\alpha$-stable closed normal subgroup $G_{\mathrm{td}}$ such that

$$
G=G_{e} \times G_{\mathrm{td}}
$$


internally as a topological group; moreover, $G_{e}$ is a simply connected, nilpotent real Lie group (see [15]) and hence torsion-free. Results by the authors imply that the set $\operatorname{tor}(G)$ of torsion elements is a closed subgroup of $G_{\mathrm{td}}$ and

$$
G=G_{e} \times G_{p_{1}} \times \cdots \times G_{p_{n}} \times \operatorname{tor}(G)
$$

internally for $\alpha$-stable closed subgroups $G_{p}$ of $G$ which are $p$-adic Lie groups for some prime $p$ (cf. [7, Theorem B]). We improve these results by adding uniqueness statements, invariance properties and refined decompositions: 1

Theorem A. For each locally compact contraction group $(G, \alpha)$, we have:

(a) $G$ has a unique closed normal subgroup $G_{\mathrm{td}}$ such that $G=G_{e} \times G_{\mathrm{td}}$ internally as a topological group. The subgroup $G_{\mathrm{td}}$ is totally disconnected, $\alpha$-stable, and topologically fully invariant in $G$.

(b) The set $\operatorname{tor}(G)$ of torsion elements of $G$ is a closed subgroup of $G$ and totally disconnected. There are a unique $n \in \mathbb{N}_{0}$, unique prime numbers $p_{1}<\cdots<p_{n}$ and unique $p$-adic Lie groups $G_{p} \neq\{e\}$ for $p \in\left\{p_{1}, \ldots, p_{n}\right\}$ which are closed normal subgroups of $G$ such that

$$
G=G_{e} \times G_{p_{1}} \times \cdots \times G_{p_{n}} \times \operatorname{tor}(G)
$$

internally as a topological group. Each $G_{p}$ is topologically fully invariant in $G$ (and hence $\alpha$-stable).

(c) If $\operatorname{tor}(G)$ is locally pro-nilpotent, then, for each prime number $p$, the set $\operatorname{tor}_{p}(G)$ of $p$-torsion elements of $G$ is a fully invariant closed subgroup of $G$ which is locally pro-p. Moreover, $\operatorname{tor}_{p}(G) \neq\{e\}$ for only finitely many $p$, say for $p$ among the prime numbers $q_{1}<q_{2}<\cdots<q_{m}$, and

$$
\operatorname{tor}(G)=\operatorname{tor}_{q_{1}}(G) \times \cdots \times \operatorname{tor}_{q_{m}}(G)
$$

internally as a topological group.

The structure of $G_{e}$ and the $G_{p}$ is well understood: Siebert showed that the Lie algebra of the simply connected nilpotent real Lie group $G_{e}$ admits a positive graduation (and any real Lie algebra with this property arises in this way), see [15]. J. S. P. Wang showed that $G_{p}$ is the group of $\mathbb{Q}_{p}$-rational points

\footnotetext{
${ }^{1}$ In the following theorems, we use terminology as explained in Section 1 .
} 
of a unipotent algebraic group defined over the field $\mathbb{Q}_{p}$ of $p$-adic numbers (and hence nilpotent) [17]; its Lie algebra admits an $\mathbb{N}$-graduation (and any $p$-adic Lie algebra with this property arises in this way; see also [6]). By contrast, the structure of the torsion part $\operatorname{tor}(G)$ is as yet not well understood, except that, as a locally compact contraction group, $\operatorname{tor}(G)$ has a composition series whose simple quotients are isomorphic to restricted products

$$
F^{(-\mathbb{N})} \times F^{\mathbb{N}_{0}}
$$

with the right shift for $F$ a finite simple group, where $F^{\mathbb{N}_{0}}$ is equipped with the compact product topology and where the group $F^{(-\mathbb{N})}$ of finitely supported sequences $\left(x_{n}\right)_{n \in-\mathbb{N}}$ in $F$ (viz. $x_{n}=e$ for all sufficiently small $n$ ) is given the discrete topology (see [7]). Note that $F^{(-\mathbb{N})} \times F^{\mathbb{N}_{0}}$ is isomorphic to the additive group of the field $\mathbb{F}_{p}((t))$ of formal Laurent series with coefficients in $\mathbb{F}_{p}$ (together with the contractive automorphism $\alpha: f(t) \mapsto t f(t)$ ) if $F:=$ $\mathbb{F}_{p}$ is a cyclic group of prime order $p$. As there are (up to isomorphism) only countably many possible composition factors, it is natural to ask whether, up to isomorphism, there are only countably many torsion contraction groups. We show that the answer is negative (see Theorem 8.1):

Theorem B. For each prime $p$, there is an uncountable set of contraction groups $(H, \beta)$ which are pairwise non-isomorphic as contraction groups and such that each $(H, \beta)$ is a central extension

$$
\{0\} \rightarrow \mathbb{F}_{p}((t)) \rightarrow H \rightarrow \mathbb{F}_{p}((t)) \rightarrow\{0\}
$$

To this end, we describe continuous 2-cocycles $\eta_{s}: \mathbb{F}_{p}((t)) \times \mathbb{F}_{p}((t)) \rightarrow \mathbb{F}_{p}((t))$ with $\alpha \circ \eta_{s}=\eta_{s} \circ(\alpha \times \alpha)$, parametrized by sequences $s \in\{0,1\}^{\mathbb{N}}$, which give rise to contraction groups

$$
\mathbb{F}_{p}((t)) \times_{\eta_{s}} \mathbb{F}_{p}((t))
$$

which are pairwise non-isomorphic for different values of $s$. This procedure is quite natural, since all central extensions of locally compact contraction groups can be described by equivariant, continuous 2-cocycles (as observed in Appendix (A), as a consequence of the following result: 
Theorem C. If $(G, \alpha)$ and $(H, \beta)$ are locally compact contraction groups and $q: G \rightarrow H$ is a surjective, continuous homomorphism such that $\beta \circ q=q \circ \alpha$, then there exists a continuous map $\sigma: H \rightarrow G$ such that $\sigma(e)=e, q \circ \sigma=\operatorname{id}_{H}$ and $\alpha \circ \sigma=\sigma \circ \beta$.

In other words, all extensions of locally compact contraction groups admit equivariant, continuous global sections.

Theorem B provides uncountably many non-isomorphic contraction groups which are central extensions of the abelian group $\mathbb{F}_{p}((t))$ by itself, and hence nilpotent. By contrast, there are only two non-isomorphic abelian contraction groups which are extensions of $\mathbb{F}_{p}((t))$ by itself. More generally, we have the following classification (which subsumes the preceding assertion with $F=$ $\mathbb{Z} / 4 \mathbb{Z}$ and the Klein 4 -group $\left.F=(\mathbb{Z} / 2 \mathbb{Z})^{2}\right)$ :

Theorem D. Let $(G, \alpha)$ be a totally disconnected, locally compact contraction group such that $G$ is a torsion group and abelian. Then $(G, \alpha)$ is isomorphic to $F^{(-\mathbb{N})} \times F^{\mathbb{N}_{0}}$ with the right shift for some finite abelian group $F$, which is determined up to isomorphism. Conversely, every such contraction group is an abelian torsion group.

Notably, up to isomorphism there are only countably many locally compact abelian contraction groups which are torsion groups (see also Corollary 5.6).

Based on Theorem D, we obtain a classification of the locally compact abelian contraction groups. To formulate it, let $\mathbb{P}$ be the set of prime numbers and $\mathbb{Q}_{\infty}:=\mathbb{R}$. For $p \in \mathbb{P} \cup\{\infty\}$, let $\overline{\mathbb{Q}_{p}}$ be an algebraic closure of $\mathbb{Q}_{p}$ and $\Omega_{p}$ be the set of all monic irreducible polynomials $f$ over $\mathbb{Q}_{p}$ such that $|\lambda|<1$ for all zeros $\lambda$ of $f$ in $\overline{\mathbb{Q}_{p}}$, where $|$.$| is the unique extension of the usual$ absolute value on $\mathbb{Q}_{p}$ to an absolute value on $\overline{\mathbb{Q}_{p}}$. Given a monic irreducible polynomial $f \in \mathbb{Q}_{p}[X]$ and $n \in \mathbb{N}$, we endow the $\mathbb{Q}_{p}$-vector space

$$
E_{f^{n}}:=\mathbb{Q}_{p}[X] / f^{n} \mathbb{Q}_{p}[X]
$$

with the $\mathbb{Q}_{p}$-linear automorphism $\alpha_{f^{n}}$ taking $g+f^{n} \mathbb{Q}_{p}[X]$ to $X g+f^{n} \mathbb{Q}_{p}[X]$ for all polynomials $g \in \mathbb{Q}_{p}[X]$ (whose characteristic and minimal polynomials both are $\left.f^{n}\right)$. Given $p \in \mathbb{P}$ and $n \in \mathbb{N}$, let $F_{p^{n}}:=\mathbb{Z} / p^{n} \mathbb{Z}$ and write $F_{p^{n}}((t))$ as a shorthand for $F_{p^{n}}^{(-\mathbb{N})} \times F_{p^{n}}^{\mathbb{N} o}$, endowed with the right shift $\alpha_{p^{n}}$ (see also 1.3).

We obtain the following Structure Theorem for Locally Compact Abelian Contraction Groups. 
Theorem E. Let $(G,+)$ be a locally compact abelian group and $\alpha: G \rightarrow G$ be a contractive automorphism. Then $(G, \alpha)$ is isomorphic to

$$
\bigoplus_{p \in \mathbb{P} \cup\{\infty\}} \bigoplus_{f \in \Omega_{p}} \bigoplus_{n \in \mathbb{N}}\left(E_{f^{n}}, \alpha_{f^{n}}\right)^{\mu(p, f, n)} \oplus \bigoplus_{p \in \mathbb{P}} \bigoplus_{n \in \mathbb{N}}\left(F_{p^{n}}((t)), \alpha_{p^{n}}\right)^{\nu(p, n)}
$$

as a contraction group, for uniquely determined $\mu(p, f, n) \in \mathbb{N}_{0}$ which are non-zero for only finitely many $(p, f, n)$ with $(p, n) \in(\mathbb{P} \cup\{\infty\}) \times \mathbb{N}$ and $f \in \Omega_{p}$, and uniquely determined $\nu(p, n) \in \mathbb{N}_{0}$ which are non-zero for only finitely many $(p, n) \in \mathbb{P} \times \mathbb{N}$. Conversely, all groups of the form $(*)$ are locally compact abelian contraction groups.

We are also able to adapt various results to general locally compact contraction groups $(G, \alpha)$ which were previously only known for totally disconnected $G$. For example, generalizing [7, Theorem 3.3] we show that every locally compact contraction group admits a composition series

$$
\{e\}=G_{0} \triangleleft G_{1} \triangleleft \cdots \triangleleft G_{n}=G
$$

as a contraction group, and that a Jordan-Hölder Theorem holds for such (see Theorem 4.7). As the composition factors $G_{j} / G_{j-1}$ are simple contraction groups (in the sense of 1.6), it is of interest to classify the latter. A classification of the simple totally disconnected contraction groups was obtained in [7, Theorem A]. The following result completes the picture:

Theorem F. If $(G, \alpha)$ is a simple locally compact contraction group, then $G$ is either connected or totally disconnected. The family $\left(E_{f}, \alpha_{f}\right)_{f \in \Omega_{\infty}}$ is a system of representatives for the isomorphism classes of the connected simple locally compact contraction groups.

It is known from [7, Corollary 3.6] that a continuous group homomorphism $\phi: G \rightarrow H$ has closed image and is an open mapping onto its image whenever $(G, \alpha)$ and $(H, \beta)$ are totally disconnected, locally compact contraction groups and $\beta \circ \phi=\phi \circ \alpha$. We obtain the following generalization (see Proposition 4.1 and Corollary 4.3):

Theorem G. Let $(G, \alpha)$ and $(H, \beta)$ be locally compact contraction groups and $\phi: G \rightarrow H$ be a continuous group homomorphism. Then we have:

(a) $\phi(G)$ is closed in $H$ if and only if $\phi(\operatorname{tor}(G))$ is closed in $H$, in which case the co-restriction $\left.\phi\right|^{\phi(G)}: G \rightarrow \phi(G)$ is an open map. 
(b) If $\beta \circ \phi=\phi \circ \alpha$, then $\phi(G)$ is closed in $H$.

Structure of the article. In Section 1, we fix notation and describe basic facts and conventions for later use. Section 2 is devoted to uniqueness properties and Theorem A. Section 3 contains the proof of Theorem C. As already mentioned, the latter theorem allows central extensions of locally compact contraction groups to be described in terms of suitably-defined equivariant continuous 2-cocycles (and likewise for extensions with abelian kernel). The arguments, which closely parallel the classical case of group extensions, are sketched in Appendix A. Theorems F and G are established in Section 4. In Section 5, we prove Theorems D and E. Section 6 provides two auxiliary results concerning extensions of contraction groups. In Section 7. we turn to a special class of 2-cocycles on contraction groups leading to central extensions, namely equivariant, biadditive, continuous mappings $\omega: \mathbb{F}_{p}((t)) \times \mathbb{F}_{p}((t)) \rightarrow \mathbb{F}_{p}((t))$. We obtain an explicit parametrization of these in terms of two-sided sequences of elements in $\mathbb{F}_{p}((t))$. In Section 8, we show that a suitable uncountable set of parameters gives rise to extensions which are not only inequivalent extensions, but pairwise non-isomorphic as contraction groups (thus proving Theorem B). The article closes with open problems concerning torsion contraction groups which are locally pro- $p$ (Section 9).

Acknowledgements. The first author is grateful to George A. Willis and the University of Newcastle, N.S.W. for support which enabled research visits in October 2015 and September 2017. Partial support also came from the Deutsche Forschungsgemeinschaft, DFG grant GL 357/10-1.

\section{Preliminaries and notation}

We write $\mathbb{Z}$ for the ring of integers, $\mathbb{N}:=\{1,2, \ldots\}$ and $\mathbb{N}_{0}:=\mathbb{N} \cup\{0\}$. If $f: X \rightarrow X$ is a self-map of a set $X$, then a subset $M \subseteq X$ is called $\alpha$-stable if $f(M)=M$. If $M$ is a subset of a group $G$ and $\alpha(M) \subseteq M$ for each endomorphism $\alpha$ of $G$, then $M$ is called fully invariant. If $G$ is a topological group and $\alpha(M) \subseteq M$ for each continuous endomorphism $\alpha$ of $G$, then $M$ is called topologically fully invariant. As usual, we write $\langle M\rangle$ for the subgroup generated by a subset $M$ of a group $G$. If $A$ and $B$ are subgroups of a group $G$, we write $[A, B]$ for the subgroup of $G$ generated by $\left\{a b a^{-1} b^{-1}: a \in A, b \in B\right\}$, as usual. We define $C^{1}(G):=G$ and $C^{n+1}(G):=\left[G, C^{n}(G)\right]$ for $n \in \mathbb{N}$. We define $G^{(0)}:=G$ and $G^{(n+1)}:=\left[G^{(n)}, G^{(n)}\right]$ for $n \in \mathbb{N}_{0}$. 
1.1 If $G$ is a group and $p$ a prime number, we write

$$
\operatorname{tor}_{p}(G):=\left\{g \in G:(\exists n \in \mathbb{N}) g^{p^{n}}=e\right\}
$$

for the subset of $p$-torsion elements. If $\operatorname{tor}_{p}(G)$ is a subgroup, we call it the p-torsion subgroup of $G$.

1.2 If $G$ and $H$ are groups and $\phi: G \rightarrow H$ is a group homomorphism, then

$$
\phi\left(\operatorname{tor}_{p}(G)\right) \subseteq \operatorname{tor}_{p}(H)
$$

for each prime number $p$. In particular, $\operatorname{tor}_{p}(G)$ is fully invariant in $G$.

1.3 If $p$ is a prime number, we let $\mathbb{F}_{p}$ be the finite field of order $p$ and $\mathbb{F}_{p}((t))$ be the valued field of formal Laurent series with coefficients in $\mathbb{F}_{p}$ (cf. [13], [18]); its elements are series of the form $x=\sum_{n=N}^{\infty} x_{n} t^{n}$ with $N \in \mathbb{Z}$ and $x_{n} \in \mathbb{F}_{p}$ for integers $n \geq N$. We shall use the absolute value $|$.$| on \mathbb{F}_{p}((t))$ given by

$$
|x|:=p^{-N}
$$

if $x \neq 0$ and $N$ (as above) is chosen minimal with $x_{N} \neq 0$. Then

$$
|x+y|=|x| \quad \text { for all } x, y \in \mathbb{F}_{p}((t)) \text { such that }|y|<|x| \text {. }
$$

In the same way, we can consider the ring $F((t))$ of formal Laurent series over a finite commutative ring $F$. Its additive group with the contractive automorphism $x \mapsto t x$ is isomorphic to $F^{(-\mathbb{N})} \times F^{\mathbb{N}_{0}}$ with the right shift, and we appreciate the more compact notation.

All topological groups we consider are assumed Hausdorff. As usual, we use locally compact group as a shorthand for locally compact topological group.

1.4 If we speak of an automorphism $\alpha$ of a locally compact group $A$, then $\alpha$ and its inverse are assumed continuous. We write $\operatorname{Aut}(A)$ for the group of all such automorphisms, and endow it with the topology induced from the Braconnier topology on the set $\operatorname{Homeo}(A)$ of auto-homeomorphisms of $A$ (called the 'refined compact-open topology' in [16]). A subbasis for this topology is given by the sets

$$
\left\{\alpha \in \operatorname{Aut}(A): \alpha\left(K_{1}\right) \subseteq U_{1} \text { and } \alpha^{-1}\left(K_{2}\right) \subseteq U_{2}\right\},
$$

for $K_{1}, K_{2}$ in the set of compact subsets of $A$ and open subsets $U_{1}, U_{2} \subseteq A$. 
1.5 If $G$ is a topological group, then a homomorphism $\gamma: G \rightarrow \operatorname{Aut}(A)$ is continuous if and only if the corresponding left $G$-action

$$
G \times A \rightarrow A, \quad(g, a) \mapsto \gamma(g)(a)
$$

is continuous (see, e.g., [16, Lemma 10.4]).

1.6 An automorphism $\alpha$ of a topological group $G$ is called contractive if

$$
\lim _{n \rightarrow \infty} \alpha^{n}(g)=e \quad \text { for all } g \in G .
$$

A locally compact contraction group is a pair $(G, \alpha)$ consisting of a locally compact group $G$ and a contractive automorphism $\alpha: G \rightarrow G$. If $G$ is, moreover, totally disconnected (resp., connected), then $(G, \alpha)$ is called a totally disconnected (resp., connected) locally compact contraction group. We call $(G, \alpha)$ a simple contraction group if $G \neq\{e\}$ and $G$ does not have $\alpha$-stable, closed normal subgroups except for $\{e\}$ and $G$.

1.7 Every contractive automorphism $\alpha$ of a locally compact group $G$ is compactly contractive in the following sense: For each compact set $K \subseteq G$ and identity neighbourhood $U \subseteq G$, there exists $N \in \mathbb{N}$ such that

$$
\alpha^{n}(K) \subseteq U \text { for all integers } n \geq N
$$

(see [17, Proposition 2.1] or [15, Lemma 1.4(iv)]). Thus $\left\{\alpha^{n}(V): n \in \mathbb{N}_{0}\right\}$ is a basis of identity neighbourhoods in $G$, for each compact identity neighbourhood $V \subseteq G$.

1.8 If $(G, \alpha)$ is a totally disconnected, locally compact contraction group, then there exists a compact open subgroup $V \subseteq G$ such that $\alpha(V) \subseteq V$ (see [15, Lemma $3.2(\mathrm{i})])$.

1.9 If $G$ is a locally compact group with Haar measure $\lambda_{G}$ and $\alpha: G \rightarrow G$ an automorphism, there is $\Delta(\alpha) \in] 0, \infty[$ (the module of $\alpha$ ) such that $\lambda_{G}(\alpha(A))=\Delta(\alpha) \lambda_{G}(A)$ for all Borel sets $A \subseteq G$ (cf. [8]). If $G$ is totally disconnected and $\alpha$ is contractive, let $V \subseteq G$ be a compact open subgroup such that $\alpha(V) \subseteq V$ (see 1.8). Then

$$
\Delta\left(\alpha^{-1}\right)=[V: \alpha(V)]
$$


1.10 Let $\left(G, \alpha_{G}\right)$ and $\left(H, \alpha_{H}\right)$ be locally compact contraction groups. A continuous group homomorphism $\phi: G \rightarrow H$ is called a morphism of contraction groups if it is equivariant in the sense that

$$
\alpha_{H} \circ \phi=\phi \circ \alpha_{G}
$$

we then write $\phi:\left(G, \alpha_{G}\right) \rightarrow\left(H, \alpha_{H}\right)$. If, moreover, $\phi$ is invertible and also $\phi^{-1}$ is a morphism of contraction groups, then $\phi$ is an isomorphism of contraction groups (and an automorphism of the contraction group $\left(G, \alpha_{G}\right)$ if, moreover, $\left.\left(G, \alpha_{G}\right)=\left(H, \alpha_{H}\right)\right)$. We write $\operatorname{Aut}\left(G, \alpha_{G}\right)$ for the group of automorphisms of $\left(G, \alpha_{G}\right)$.

1.11 If $\phi:\left(G, \alpha_{G}\right) \rightarrow\left(H, \alpha_{H}\right)$ is a morphism between totally disconnected, locally compact contraction groups, then $\phi(G)$ is a closed, $\alpha_{H}$-stable subgroup of $H$ and the co-restriction $\left.\phi\right|^{\phi(G)}: G \rightarrow \phi(G)$ is a quotient homomorphism (i.e., surjective, continuous, and open), see [7, Corollary 3.6].

1.12 If $\left(A, \alpha_{A}\right),(\widehat{G}, \widehat{\alpha})$, and $(G, \alpha)$ are totally disconnected, locally compact contraction groups, we call a short exact sequence

$$
\{e\} \rightarrow A \stackrel{\iota}{\rightarrow} \widehat{G} \stackrel{q}{\rightarrow} G \rightarrow\{e\}
$$

(or simply $\widehat{G})$ an extension of contraction groups if $\iota:\left(A, \alpha_{A}\right) \rightarrow(\widehat{G}, \widehat{\alpha})$ and $q:(\widehat{G}, \widehat{\alpha}) \rightarrow(G, \alpha)$ are morphisms of contraction groups. Then $\iota$ is a homeomorphism onto its image and $q$ is a quotient homomorphism, by the fact recalled in 1.11. If $A$ is abelian, then $\widehat{G}$ is called an extension of $G$ with abelian kernel; if $\iota(A)$ is contained in the centre $Z(\widehat{G})$ of the group $\widehat{G}$, then $\widehat{G}$ is called a central extension of $G$.

1.13 If $(G, \alpha)$ is a locally compact contraction group, we call a series

$$
\{e\} \triangleleft G_{0} \triangleleft G_{1} \triangleleft \cdots \triangleleft G_{n}=G
$$

a series of contraction groups 2 if $G_{j}$ is a closed $\alpha$-stable subgroup of $G$ for each $j \in\{0, \ldots, n\}$. We endow $G_{j}$ with the contractive automorphism $\left.\alpha\right|_{G_{j}}$ for $j \in$ $\{0, \ldots, n\}$. Moreover, we endow $G_{j} / G_{j-1}$ with the contractive automorphism $\bar{\alpha}_{j}: x G_{j-1} \mapsto \alpha(x) G_{j-1}$ for $j \in\{1, \ldots, n\}$. If $\left(G_{j} / G_{j-1}, \bar{\alpha}_{j}\right)$ is a simple contraction group for all $j \in\{1, \ldots, n\}$, then (5) is called a composition series of contraction groups.

\footnotetext{
${ }^{2}$ In the terminology of [7, Definition 2.1], this is an $\langle\alpha\rangle$-series.
} 
1.14 Following [15, Definition 1.7], we say that a locally compact group $G$ is contractible if it admits a contractive automorphism $\alpha: G \rightarrow G$.

Repeatedly, we shall construct continuous functions as follows.

Lemma 1.15 Let $X$ be a topological space, $G$ be a totally disconnected, locally compact group which is metrizable and $\left(f_{n}\right)_{n \in \mathbb{N}_{0}}$ be a sequence of continuous functions $f_{n}: X \rightarrow G$. Assume that there exists a basis of identity neighbourhoods $U_{1} \supseteq U_{2} \supseteq \cdots$ in $G$ such that $U_{n}$ is a compact open subgroup of $G$ and $f_{n}(X) \subseteq U_{n}$, for each $n \in \mathbb{N}$. Then

$$
g_{n}:=f_{0} f_{1} \ldots f_{n}: X \rightarrow G, \quad x \mapsto f_{0}(x) f_{1}(x) \cdots f_{n}(x)
$$

converges uniformly to a continuous function $g: X \rightarrow G$.

Proof. For all $n \in \mathbb{N}_{0}$ and $m \in \mathbb{N}_{0}$, we have

$$
\left(g_{n+m}(x)\right)^{-1} g_{n}(x)=\left(f_{n+m}(x)\right)^{-1} \cdots\left(f_{n+1}(x)\right)^{-1} \in U_{n+1} \text { for all } x \in X .
$$

For fixed $x \in X$, we deduce that $\left(g_{n}(x)\right)_{n \in \mathbb{N}}$ is a Cauchy sequence in $G$ and hence convergent (as locally compact groups are complete). Let $g(x)$ be the limit. Letting $m \rightarrow \infty$ in ([6), we see that

$$
(g(x))^{-1} g_{n}(x) \in U_{n+1} \text { for all } n \in \mathbb{N}_{0} \text { and } x \in X .
$$

Thus $g_{n} \rightarrow g$ uniformly, whence $g$ is continuous.

1.16 Let $\mathbb{K}=\mathbb{R}$ or $\mathbb{K}=\mathbb{Q}_{p}$, respectively, for some prime $p$. Recall that a real (resp. $p$-adic) Lie group is a group $G$, endowed with a finite-dimensional $\mathbb{K}$ analytic manifold structure which makes the group operations analytic. Then the tangent space $L(G):=T_{e}(G)$ of $G$ at the neutral element is a Lie algebra over $\mathbb{K}$ in a natural way. If $\phi: G \rightarrow H$ is a continuous group homomorphism, then $\phi$ is analytic and we write $L(\phi):=T_{e}(\phi)$ for its tangent map at $e$, which is a Lie algebra homomorphism from $L(G)$ to $L(H)$. In particular, there is at most one real (resp., $p$-adic) Lie group structure on a given locally compact group $G$. As usual, we identify a real (resp. p-adic) Lie group with its underlying locally compact group (see [2] and [14] for further information).

For generalities concerning pro-finite groups and pro- $p$-groups, the reader is referred to [19] and [4]. 
Definition 1.17 Let $p$ be a prime. We say that a locally compact group $G$ is locally pro- $p$ if it has a compact open subgroup $U$ which is a pro-p-group.

For example, every $p$-adic Lie group is locally pro- $p$ (cf. [4, Theorems 8.29 and 8.31]).

Definition 1.18 We say that a locally compact group $G$ is locally pronilpotent if it has a compact open subgroup $U$ which is pro-nilpotent (i.e., a projective limit of finite nilpotent groups).

For example, every totally disconnected, abelian locally compact contraction group $(G, \alpha)$ is locally pro-nilpotent, as every compact open subgroup $U \subseteq G$ is abelian and hence pro-nilpotent.

\section{Decompositions of contraction groups}

In this section, we prove Theorem A and provide additional results concerning decompositions of locally compact contraction groups.

We first pinpoint properties of group elements in special classes of contraction groups (like totally disconnected groups, connected groups, $p$-adic Lie groups, and torsion groups). These properties will help us to distinguish elements in the individual factors of decompositions of contraction groups, and hence to see that the factors are unique.

\section{Divisible elements; topologically periodic elements}

2.1 Recall that an element $g$ in a group $G$ is called divisible if, for each $n \in \mathbb{N}$, there exists $x \in G$ such that $x^{n}=g$. If $x$ is uniquely determined for each $n$, then $g$ is called uniquely divisible. An element $g$ of a locally compact group $G$ is called topologically periodic if $g$ generates a relatively compact subgroup. If $\phi: G \rightarrow H$ is a continuous homomorphism between locally compact groups and $g \in G$ is topologically periodic, then $\phi(g)$ is topologically periodic in $H$.

Lemma 2.2 If $(G, \alpha)$ is a totally disconnected, locally compact contraction group, then $G$ is a union of compact open subgroups. In particular, each $g \in G$ is topologically periodic.

Proof. Let $V$ be a compact open subgroup of $G$. Then $G=\bigcup_{n \in \mathbb{N}_{0}} \alpha^{-n}(V)$, from which the assertions follow. 


\section{Basic properties of connected contraction groups}

Results by Siebert [15] imply:

Lemma 2.3 If $(G, \alpha)$ is a connected locally compact contraction group, then $G$ is a simply connected, nilpotent real Lie group, $L(G)$ is a nilpotent real Lie algebra, $L(\alpha): L(G) \rightarrow L(G)$ is contractive and we have:

(a) $(G, \alpha)$ is isomorphic to $((L(G), *), L(\alpha))$ as a contraction group, where $*: L(G) \times L(G) \rightarrow L(G),(x, y) \mapsto x * y=x+y+\frac{1}{2}[x, y]+\cdots$ is the Baker-Campbell-Hausdorff (BCH)- multiplication.

(b) $G$ is torsion-free and each $g \in G$ is uniquely divisible.

(c) If $g \in G$ is topologically periodic, then $g=e$.

(d) For each $e \neq g \in G$ and each sequence $\left(k_{n}\right)_{n \in \mathbb{N}}$ in $\mathbb{N}$ such that $k_{n} \rightarrow \infty$, the sequence $\left(g^{k_{n}}\right)_{n \in \mathbb{N}}$ does not converge in $G$.

Proof. By [15, 2.3 and Corollary 2.4], $G$ is a simply connected real Lie group whose Lie algebra $L(G)$ is nilpotent. Hence $G$ is nilpotent (cf. Proposition 12 in [2, Chapter III, §9.5]). Since $L(G)$ is nilpotent, the BCH-series converges on all of $L(G) \times L(G)$ and makes $L(G)$ a Lie group with neutral element 0 (see explanations after Proposition 12 in [2, Chapter III, §9.5]). Since $G$ is simply connected and nilpotent, the exponential map $\exp _{G}: L(G) \rightarrow G$ is an isomorphism of Lie groups from $(L(G), *)$ to $G$, by Proposition 13 in $[2$, Chapter III, §9.5]. Since

$$
\exp _{G} \circ L(\alpha)=\alpha \circ \exp _{G}
$$

by Proposition 10 in [2, Chapter III, §6.4], we see that the automorphism $L(\alpha): L(G) \rightarrow L(G)$ of $(L(G), *)$ (and of $(L(G),+))$ is contractive. By (7), $\exp _{G}$ is an isomorphism of contraction groups from $((L(G), *), L(\alpha))$ to $(G, \alpha)$, establishing (a).

Using the BCH-formula, for $x \in L(G)$ we find that the map $\mathbb{R} \rightarrow L(G)$, $t \mapsto t x$ is a continuous group homomorphism to $(L(G), *)$, whence $\mathbb{R} x$ is a subgroup of $(L(G), *)$ and

$$
x^{n}=n x \quad \text { for all } n \in \mathbb{Z} .
$$

Since $(L(G),+)$ is torsion-free and each element of this group is uniquely divisible, we deduce with (8) that also the group $(L(G), *)$ is torsion-free and 
each of its elements is uniquely divisible (entailing (b)). If $0 \neq x \in L(G)$ was topologically periodic in $(L(G), *)$, then the discrete group $\langle x\rangle=\mathbb{Z} x \cong \mathbb{Z}$ would be relatively compact in $\mathbb{R} x \cong \mathbb{R}$, a contradiction. Thus (c) holds.

To establish (d), let $\|$.$\| be a norm on L(G)$. If $0 \neq x \in L(G)$ and $k_{n} \rightarrow \infty$, then $\left\|x^{k_{n}}\right\|=\left\|k_{n} x\right\|=k_{n}\|x\| \rightarrow \infty$, whence $x^{k_{n}}$ cannot converge in $L(G)$.

\section{Basic properties of $\boldsymbol{p}$-adic contraction groups}

For $p$-adic contraction groups, a counterpart of Lemma 2.3 is available.

Lemma 2.4 If $(G, \alpha)$ is a locally compact contraction group such that $G$ is a p-adic Lie group, then $G$ is nilpotent, $L(G)$ is a nilpotent p-adic Lie algebra, $L(\alpha): L(G) \rightarrow L(G)$ is contractive and we have:

(a) $(G, \alpha)$ is isomorphic to $((L(G), *), L(\alpha))$ as a contraction group, where *: $L(G) \times L(G) \rightarrow L(G)$ is the BCH-multiplication.

(b) $G$ is torsion-free and each $g \in G$ is uniquely divisible.

(c) For each $g \in G$, we have $\lim _{n \rightarrow \infty} g^{p^{n}}=e$.

Proof. By [17, Theorem 3.5 (ii) and (iv)], $G$ is nilpotent and $L(\alpha)$ is contractive. Hence $L(G)$ is a nilpotent $p$-adic Lie algebra (by Proposition 12 in [2, Chapter III, §9.4]), ensuring that the BCH-series converges on all of $L(G) \times L(G)$ and makes $L(G)$ a $p$-adic Lie group (see explanations after Proposition 12 in [2, Chapter III, §9.5]). The Lie algebra automorphism $L(\alpha)$ also is an automorphism of $(L(G), *$ ) (as the BCH-series is given by nested Lie brackets). There exists a compact open subgroup $V$ of $(L(G), *)$ and an exponential function $\exp _{G}: V \rightarrow G$ whose image $U$ is a compact open subgroup of $G$, such that $\exp _{G}:(V, *) \rightarrow U$ is an isomorphism of $p$-adic Lie groups, and such that

$$
\left.\exp _{G} \circ L(\alpha)\right|_{W}=\left.\alpha \circ \exp _{G}\right|_{W}
$$

for some compact open subgroup $W$ of $(V, *)$ such that $L(\alpha)(W) \subseteq V$ (cf. Proposition 8 in [2, Chapter III, §4.4]). Since $L(\alpha)$ is contractive, there exists a compact open subgroup $Q$ of $(L(G)$, *) such that $Q \subseteq W$ and $L(\alpha)(Q) \subseteq Q$ (cf. 1.8 and 1.7); after replacing both $V$ and $W$ with $Q$, we may assume that $L(\alpha)(V) \subseteq V$ and $\left.\exp _{G} \circ L(\alpha)\right|_{V}=\alpha \circ \exp _{G}$. Now [17, Proposition 2.2] provides an isomorphism $\phi:((L(G), *), L(\alpha)) \rightarrow(G, \alpha)$ of contraction groups 
which extends $\exp _{G}$ (establishing (a)).

Since $\mathbb{Q}_{p} \rightarrow L(G), t \mapsto t x$ is a continuous homomorphism to $(L(G), *)$ for each $x \in L(G)$, we see that (8) holds and deduce as in the proof of Lemma 2.3 that $(L(G), *)$ is torsion-free and all of its elements are uniquely divisible (as both properties are inherited from $(L(G),+))$. Thus (b) holds.

Using (8), we see that $x^{p^{n}}=p^{n} x \rightarrow 0$ as $n \rightarrow \infty$ for each element $x$ of $(L(G), *)$, from which (c) follows.

Lemma 2.5 Let $p \neq q$ be prime numbers, $G$ be a torsion-free $p$-adic Lie group (e.g., a contractible p-adic Lie group), and $e \neq g \in G$. Then the sequence $\left(g^{q^{n}}\right)_{n \in \mathbb{N}}$ does not converge to e in $G$.

Proof. There exists an open 0-neighbourhood $V \subseteq L(G)$ such that the $\mathrm{BCH}$-series converges on $V \times V$ and makes $V$ a $p$-adic Lie group $(V, *)$, and an open subgroup $U \subseteq G$ which is isomorphic to $(V, *)$ (cf. Theorem 2 and Lemma 3 in [2, Chapter III, §4.2]). Let $\phi: U \rightarrow V$ be an isomorphism. If there were $g \in G \backslash\{e\}$ such that $g^{q^{n}} \rightarrow e$, then we could find $m \in \mathbb{N}$ such that $g^{q^{n}} \in U$ for all $n \geq m$. Since $G$ is torsion-free, $g^{q^{m}} \neq e$ and thus $x:=\phi\left(g^{q^{m}}\right) \neq 0$. Choose a norm $\|$.$\| on the p$-adic vector space $L(G)$. We have $x^{q^{n}}=\phi\left(g^{q^{n+m}}\right) \rightarrow 0$ as $n \rightarrow \infty$. But $q$ has $p$-adic absolute value $|q|_{p}=1$, as is well-known 3 Hence $\left\|x^{q^{n}}\right\|=\left\|q^{n} x\right\|=|q|_{p}^{n}\|x\|=\|x\|$ for all $n \in \mathbb{N}$ and thus $x^{q^{n}} \nrightarrow 0$, contradiction.

\section{Basic properties of torsion contraction groups}

Lemma 2.6 Let $(G, \alpha)$ be a locally compact contraction group and tor $(G)$ be the set of all torsion elements of $G$. Then $\operatorname{tor}(G)$ is a fully invariant closed subgroup of $G$ and totally disconnected.

Proof. By [15, Proposition 4.2], $G$ has a totally disconnected, $\alpha$-stable closed normal subgroup $D$ such that $G=G_{e} \times D$ internally as a topological group. Since $G_{e}$ is torsion-free by Lemma 2.3 , we have $\operatorname{tor}(G)=\operatorname{tor}(D)$. As $\operatorname{tor}(G)=\operatorname{tor}(D)$ is a closed subgroup of $D$ by [7, Theorem B], it also is a closed subgroup of $G$ (and fully invariant by a trivial argument).

\footnotetext{
${ }^{3}$ We have $a p+b q=1$ for suitable integers $a$ and $b$, whence $b q=1-a p$ and hence $|b|_{p}|q|_{p}=1$. Since $|b|_{p},|q|_{p} \leq 1$, the latter entails $|q|_{p}=1$.
} 
Lemma 2.7 If $(G, \alpha)$ is a locally compact contraction group and $G$ is a torsion group, then $G$ is totally disconnected and has finite exponent. If an element $g \in G$ is divisible, then $g=e$.

Proof. By Lemma 2.6, $G$ is totally disconnected. The torsion group $G$ has finite exponent, as there exists a composition series $\{e\}=G_{0} \triangleleft G_{1} \triangleleft \cdots \triangleleft G_{n}=$ $G$ of closed $\alpha$-stable subgroups (see [7, Theorem 3.3]) and each of the factors $G_{j} / G_{j-1}$ is a torsion group of finite exponent (cf. [7, Theorem A (a)]). Being a torsion group of finite exponent, $G$ does not contain non-trivial divisible elements.

\section{Basic properties of contraction groups which are locally pro-p}

Lemma 2.8 Let $(G, \alpha)$ be a totally disconnected, locally compact contraction group. If $G$ is locally pro-p for some prime $p$, then every compact subgroup of $G$ is a pro-p-group; moreover, $G$ is a union of compact open subgroups which are pro-p-groups.

Proof. Let $K$ be a compact subgroup of $G$ and $U$ be a compact open subgroup of $G$ which is a pro-p-group. Since $\alpha$ is compactly contractive, we have $\alpha^{n}(K) \subseteq U$ for some $n \in \mathbb{N}$, whence $\alpha^{n}(K)$ is a pro- $p$-group by [4, Proposition 1.11(i)]. Since $\left.\alpha^{n}\right|_{K}: K \rightarrow \alpha^{n}(K)$ is an isomorphism, also $K$ is a pro-p-group. By Lemma 2.2 , $G$ is a union of compact open subgroups; any such is a pro- $p$-group, as just explained.

We shall use the following well-known fact:

Lemma 2.9 Let $p \neq q$ be primes, $P$ be a pro-p-group and $Q$ be a pro- $q$ group. If $\phi: P \rightarrow Q$ is a continuous group homomorphism, then $\phi(x)=e$ for all $x \in P$.

Proof. On the one hand, $\phi(P)$ is a pro- $p$-group, being isomorphic to $P / \operatorname{ker}(\phi)$ (see [4, Proposition 1.11 (b)]). On the other hand, $\phi(P)$ is a pro- $q$-group, being a compact subgroup of $Q$ (see [4, Proposition $1.11(\mathrm{a})]$ ). Hence $\phi(P) / U$ is both a $p$-group and a $q$-group and thus $\phi(P) / U=\{e\}$, for each open normal subgroup $U \subseteq \phi(P)$. As a consequence, $\phi(P)=\{e\}$. 


\section{Continuous group homomorphisms between contraction groups}

Lemma 2.10 Let $\phi: G \rightarrow H$ be a surjective, continuous group homomorphism between contractible locally compact groups $G$ and $H$. Then $\phi$ is an open map. If $\phi$ is bijective, then $\phi$ is a homeomorphism.

Proof. Since $G$ and $H$ are $\sigma$-compact by [15, 1.8(a)] and locally compact, the Open Mapping Theorem (e.g., [8, Theorem 5.29]) applies to $\phi$.

Lemma 2.11 Let $p \neq q$ be primes, $G$ and $H$ be contractible locally compact groups and $\phi: G \rightarrow H$ be a continuous group homomorphism. Then $\phi=e$ in each of the following cases:

(a) $G$ is connected and $H$ is a torsion group or a p-adic Lie group.

(b) $G$ is a p-adic Lie group and $H$ is a torsion group or a q-adic Lie group.

(c) $G$ is a torsion group and $H$ is a p-adic Lie group.

(d) $G$ is locally pro-p and $H$ is locally pro-q.

(e) $G$ is totally disconnected and $H$ is connected.

Proof. (a) If $H$ is a torsion group, then $H$ is totally disconnected (see 2.7), and so is every $p$-adic Lie group. As $\phi(G)$ is connected, $\phi(G)=\{e\}$ follows.

(b) If $H$ is a torsion group, we exploit that each $g \in G$ is divisible (by Lemma 2.4(b)). Hence $\phi(g)$ is divisible and thus $\phi(g)=e$, by Lemma 2.7. If $H$ is a $q$-adic Lie group, then the assertion is a special case of (d).

(c) Since $H$ is torsion-free as recalled in 2.4 while $\phi(G) \subseteq H$ is a torsion group, we must have $\phi(G)=\{e\}$.

(d) Given $g \in G$, there exists a compact open subgroup $U$ of $G$ which is a pro-p-group and contains $g$ (see Lemma 2.8). Then $\phi(U)$ is a compact subgroup of $H$ and hence a pro-q-group, by Lemma 2.8. By Lemma 2.9, $\left.\phi\right|_{U}: U \rightarrow \phi(U)$ is the constant map $e$, whence $\phi(g)=e$ in particular.

(e) Each $g \in G$ is topologically periodic (see Lemma 2.2), whence $\phi(g)$ is topologically periodic and thus $\phi(g)=e$ (see Lemma 2.3).

Lemma 2.12 Let $p \neq q$ be prime numbers and $\phi: G \rightarrow H$ be a continuous group homomorphism from a contractible p-adic Lie group to a torsion-free q-adic Lie group $H$. Then $\phi=e$. 
Proof. For each $g \in G$, we have $g^{p^{n}} \rightarrow e$ as $n \rightarrow \infty$, whence $\phi(g)^{p^{n}} \rightarrow e$ and thus $\phi(g)=e$, by Lemma 2.5](c).

Lemma 2.13 Let $\phi: G \rightarrow H$ be a continuous group homomorphism between contractible locally compact groups $G$ and $H$. Assume that

$$
G=G_{e} \times D \quad \text { and } \quad H=H_{e} \times E
$$

internally as topological groups for closed normal subgroups $D \subseteq G$ and $E \subseteq$ $H$. Then $\phi\left(G_{e}\right) \subseteq H_{e}$ and $\phi(D) \subseteq E$. If $\phi$ is surjective, then $\phi\left(G_{e}\right)=H_{e}$ and $\phi(D)=E$.

Proof. The assertion $\phi\left(G_{e}\right) \subseteq H_{e}$ is trivial. Write $\left.\phi\right|_{D}=\left(\phi_{e}, \theta\right)$ with $\phi_{e}: D \rightarrow H_{e}$ and $\theta: D \rightarrow E$. Since $D \cong G / G_{e}$ is totally disconnected and contractible, we have $\phi_{e}=e$ by Lemma $2.11(\mathrm{e})$ and thus $\phi(D) \subseteq E$. Notably, $\phi(G)=\phi\left(G_{e}\right) \times \phi(D)$, from which the final assertion follows.

Proposition 2.14 Let $(G, \alpha)$ and $(H, \beta)$ be locally compact contraction groups and $\phi: G \rightarrow H$ be a continuous group homomorphism. Assume that

$$
G=G_{e} \times G_{p_{1}} \times \cdots \times G_{p_{n}} \times \operatorname{tor}(G)
$$

internally as a topological group, for certain prime numbers $p_{1}<\cdots<p_{n}$ and non-trivial, $\alpha$-stable closed normal subgroups $G_{p} \subseteq G$ which are p-adic Lie groups, for $p \in\left\{p_{1}, \ldots, p_{n}\right\}$. Moreover, assume that

$$
H=H_{e} \times H_{q_{1}} \times \cdots \times H_{q_{m}} \times \operatorname{tor}(H)
$$

internally as a topological group, for certain prime numbers $q_{1}<\cdots<q_{m}$ and non-trivial, closed normal subgroups $H_{p} \subseteq H$ which are $p$-adic Lie groups, for all $p \in\left\{q_{1}, \ldots, q_{m}\right\}$. For primes $p$ such that $p \notin\left\{q_{1}, \ldots, q_{m}\right\}$, set $H_{p}:=\{e\}$. Then the following holds:

(a) $\phi\left(G_{e}\right) \subseteq H_{e}$ and $\phi(\operatorname{tor}(G)) \subseteq \operatorname{tor}(H)$;

(b) $\phi\left(G_{p}\right) \subseteq H_{p}$ for all $p \in\left\{p_{1}, \ldots, p_{n}\right\}$;

(c) If $\phi$ is injective, then $\left\{p_{1}, \ldots, p_{n}\right\} \subseteq\left\{q_{1}, \ldots, q_{m}\right\}$;

(d) If $\phi$ is surjective, then $\phi\left(G_{e}\right)=H_{e}, \phi(\operatorname{tor}(G))=\operatorname{tor}(H),\left\{p_{1}, \ldots, p_{n}\right\} \supseteq$ $\left\{q_{1}, \ldots, q_{m}\right\}$ and $\phi\left(G_{p}\right)=H_{p}$ for all $p \in\left\{q_{1}, \ldots, q_{m}\right\}$. 
Proof. (a) is obvious. To prove (b), note that $H_{e} \times H_{q_{1}} \times \cdots \times H_{q_{m}} \cong$ $H / \operatorname{tor}(H)$ is torsion-free. So $H_{q}$ is torsion-free for all $q \in\left\{q_{1}, \ldots, q_{m}\right\}$. Write

$$
\left.\phi\right|_{G_{p}}=\left(\phi_{e}, \phi_{1}, \ldots, \phi_{m}, \theta\right)
$$

in terms of its components $\phi_{e}: G_{p} \rightarrow H_{e}, \phi_{j}: G_{p} \rightarrow H_{q_{j}}$ for $j \in\{1, \ldots, m\}$, and $\theta: G_{p} \rightarrow \operatorname{tor}(H)$ with respect to the decomposition (10). By Lemma 2.11 (e) and (b), we have $\phi_{e}=e$ and $\theta=e$. Moreover, $\phi_{j}=e$ for all $j \in\{1, \ldots, m\}$ such that $p \neq q_{j}$, by Lemma 2.12. If $p \notin\left\{q_{1}, \ldots, q_{m}\right\}$, we deduce that $\phi\left(G_{p}\right)=\{e\}=H_{p}$. If $p=q_{j}$ for some $j \in\{1, \ldots, m\}$, we deduce that $\phi\left(G_{p}\right) \subseteq H_{p}$.

(c) is immediate from (b).

(d) For $j \in\{1, \ldots, m\}$, let $S_{j}:=\phi_{j}\left(G_{p_{i}}\right) \subseteq H_{q_{j}}$ if there exists $i \in$ $\{1, \ldots, n\}$ such that $p_{i}=q_{j}$; otherwise, we set $S_{j}:=\{e\} \subseteq H_{q_{j}}$. Then

$$
\phi(G)=\phi\left(G_{e}\right) \times S_{1} \times \cdots \times S_{m} \times \phi(\operatorname{tor}(G)),
$$

where $\phi\left(G_{e}\right) \subseteq H_{e}$ and $\phi(\operatorname{tor}(G)) \subseteq \operatorname{tor}(H)$. If $\phi$ is onto, then a comparison of (10) and (11) yields the conclusion of (d).

Proof of Theorem A. (a) By [15, Proposition 4.2], $G$ has a totally disconnected, $\alpha$-stable, closed normal subgroup $D$ such that $G=G_{e} \times D$ internally as a topological group. If also $E$ is a closed normal subgroup of $G$ such that $G=G_{e} \times E$ internally as a topological group, applying Lemma 2.13 to $\operatorname{id}_{G}: G_{e} \times D \rightarrow G_{e} \times E$ we obtain $D=E$. Again by Lemma 2.13, $D$ is topologically fully invariant.

(b) Write $G=G_{e} \times G_{\mathrm{td}}$ as in (a). By Lemma 2.6 and its proof, $\operatorname{tor}(G)=$ $\operatorname{tor}\left(G_{\mathrm{td}}\right)$ is a closed subgroup of $G$. By [7, Theorem B], there are primes $p_{1}<\cdots<p_{n}$ and $\alpha$-stable closed normal non-trivial subgroups $G_{p}$ of $G$ for $p \in\left\{p_{1}, \ldots, p_{n}\right\}$ such that (9) holds. Now assume that $q_{1}<\cdots<q_{m}$ are primes and

$$
G=G_{e} \times H_{q_{1}} \times \cdots \times H_{q_{m}} \times \operatorname{tor}(G)
$$

internally as a topological group for non-trivial $p$-adic Lie groups $H_{p}$ for $p \in\left\{q_{1}, \ldots, q_{m}\right\}$ which are closed normal subgroups of $G$. Applying Proposition 2.14 to $\phi:=\mathrm{id}_{G}$, we find that $n=m, p_{i}=q_{i}$ for all $i \in\{1, \ldots, n\}$ and $G_{p}=H_{p}$ for all $p \in\left\{p_{1}, \ldots, p_{n}\right\}$. By Proposition 2.14, each $G_{p}$ is topologically fully invariant in $G$.

(c) will be established later in this section, after Proposition 2.18,

More can be said concerning the $p$-adic Lie groups $G_{p}$ in Theorem A. 
Proposition 2.15 Let $(G, \alpha), p_{1}<\ldots<p_{n}$ and $G_{p}$ for $p \in\left\{p_{1}, \ldots, p_{n}\right\}$ be as in Theorem A. Write $G_{p}:=\{e\}$ for primes $p \notin\left\{p_{1}, \ldots, p_{n}\right\}$. Then

$$
G_{p}=\left\{x \in G: x \text { is divisible and } \lim _{n \rightarrow \infty} x^{p^{n}}=e\right\}
$$

for each prime number $p$.

Proof. Let $\pi_{e}: G \rightarrow G_{e}, \theta: G \rightarrow \operatorname{tor}(G)$ and $\pi_{p}: G \rightarrow G_{p}$ (for $p \in$ $\left.\left\{p_{1}, \ldots, p_{n}\right\}\right)$ be the projections onto the components of

$$
G=G_{e} \times G_{p_{1}} \times \cdots \times G_{p_{n}} \times \operatorname{tor}(G) .
$$

For each prime number $p$, the left-hand-side of (12) is a subset of the righthand-side, by Lemma 2.4(c). To see the converse inclusion, let $x$ be an element of the right-hand-side. Then $\theta(x)$ is a divisible element of $\operatorname{tor}(G)$ and hence $\theta(x)=e$, by Lemma 2.7. Moreover, $\pi_{e}(x)=e$ by Lemma $2.3(\mathrm{~d})$. Finally, $\pi_{q}(x)=e$ for each $q \in\left\{p_{1}, \ldots, p_{n}\right\}$ such that $q \neq p$, by Lemma 2.5. If $p \notin\left\{p_{1}, \ldots, p_{n}\right\}$, we deduce that $x=e$, whence $x \in G_{p}$. If $p \in\left\{p_{1}, \ldots, p_{n}\right\}$, we deduce that $x=\pi_{p}(x) \in G_{p}$.

\section{Locally pro-nilpotent contraction groups}

Proposition 2.16 Let $(G, \alpha)$ be a locally compact contraction group such that $G_{\mathrm{td}}$ is locally pro-nilpotent. Then $G$ has a largest $\alpha$-stable closed normal subgroup $G_{(p)}$ which is locally pro-p, for each prime number p. Moreover, $G_{(p)} \neq\{e\}$ only for finitely many primes, say for $p$ in the set $\left\{p_{1}, \ldots, p_{m}\right\}$ with $p_{1}<\cdots<p_{m}$, and

$$
G=G_{e} \times G_{\left(p_{1}\right)} \times \cdots \times G_{\left(p_{m}\right)}
$$

internally as a topological group.

Proof. Let $U \subseteq G_{\mathrm{td}}$ be a compact open subgroup which is pro-nilpotent. Let $V \subseteq G$ be a compact open subgroup such that $\alpha(V) \subseteq V$ (see 1.8). Then $\alpha^{n}(V) \subseteq U$ for some $n \in \mathbb{N}$, by [1.7. Thus $\alpha^{n}(V)$ is pro-nilpotent, and hence so is $V$. After replacing $U$ with $V$, we may assume that $U$ is pro-nilpotent and $\alpha(U) \subseteq U$. Being pro-nilpotent, $U$ has a unique $p$-Sylow subgroup $S_{p}$ for each prime $p$ (see [19, Propositions 2.4.3 (ii) and 2.2.2(d)]), and

$$
U=\prod_{p \in \mathbb{P}} S_{p}
$$


(see [19, Proposition 2.4.3(iii)]). As $\alpha$ is an automorphism, $\alpha\left(S_{p}\right)$ is the unique Sylow $p$-subgroup of $\alpha(U)$. Now $\alpha\left(S_{p}\right)$ is the intersection of a $p$-Sylow subgroup of $U$ with $\alpha(U) \subseteq U$, as follows from [19, Proposition 2.2.2 (c)]. So

$$
\alpha\left(S_{p}\right)=S_{p} \cap \alpha(U) \subseteq S_{p}
$$

By [7, Proposition 3.1], $G_{(p)}:=\bigcup_{n \in \mathbb{N}_{0}} \alpha^{-n}\left(S_{p}\right)$ is a closed subgroup of $G_{\mathrm{td}}$, and $S_{p}$ is open in $G_{(p)}$. Now $\alpha^{-n}\left(S_{p}\right)$ is normal in $\alpha^{-n}(U)$, for each $n \in \mathbb{N}_{0}$, since $S_{p}$ is normal in $U$. As a consequence, the ascending union $G_{(p)}=$ $\bigcup_{n \in \mathbb{N}_{0}} \alpha^{-n}\left(S_{p}\right)$ is normal in the ascending union $G_{\mathrm{td}}=\bigcup_{n \in \mathbb{N}_{0}} \alpha^{-n}(U)$ and hence in $G=G_{e} \times G_{\mathrm{td}}$. By (14) and (15), we have

$$
\alpha(U)=\prod_{p \in \mathbb{P}} \alpha\left(S_{p}\right)=\prod_{p \in \mathbb{P}}\left(\alpha(U) \cap S_{p}\right) .
$$

As $\alpha(U)$ is open in $U$, for all but finitely many $p$ we must have $S_{p}=\alpha(U) \cap S_{p}$ (which is compact) and thus $S_{p}=\{e\}$ (as every compact contraction group is trivial). Let $p_{1}<\cdots<p_{m}$ be the primes $p$ for which $S_{p} \neq\{e\}$. Then

$$
G_{\left(p_{1}\right)} \cdots G_{\left(p_{m}\right)}=\bigcup_{n \in \mathbb{N}_{0}} \alpha^{-n}(U)=G_{\mathrm{td}}
$$

showing that the product map

$$
\pi: G_{\left(p_{1}\right)} \times \cdots \times G_{\left(p_{m}\right)} \rightarrow G_{\mathrm{td}}, \quad\left(g_{1}, \ldots, g_{m}\right) \mapsto g_{1} g_{2} \cdots g_{m}
$$

is surjective. Now $S_{p_{j}}$ centralizes $S_{p_{k}}$ for all $j \neq k$ in $\{1, \ldots, m\}$, whence the ascending union $G_{\left(p_{j}\right)}=\bigcup_{n \in \mathbb{N}_{0}} \alpha^{-n}\left(S_{j}\right)$ centralizes the ascending union $G_{\left(p_{k}\right)}=\bigcup_{n \in \mathbb{N}_{0}} \alpha^{-n}\left(S_{p_{k}}\right)$. As a consequence, $\pi$ is a group homomorphism and hence a morphism of contraction groups if we use

$$
\left.\alpha\right|_{G_{\left(p_{1}\right)}} \times \cdots \times\left.\alpha\right|_{G_{\left(p_{m}\right)}}
$$

on the direct product. Thus $\operatorname{ker}(\pi)$ is a contraction group. As $\operatorname{ker}(\pi) \cap$ $\left(S_{p_{1}} \times \cdots \times S_{p_{m}}\right)=\{(e, \ldots, e)\}$, the contraction group $\operatorname{ker}(\pi)$ is discrete and thus $\operatorname{ker}(\pi)=\{(e, \ldots, e)\}$. Thus $\pi$ is bijective and hence an isomorphism of contraction groups, by 1.11. As a consequence, also (13) holds. For $k \in$ $\{1, \ldots, m\}$, let $\operatorname{pr}_{k}: G \rightarrow G_{\left(p_{k}\right)}$ be the projection onto the factor $G_{\left(p_{k}\right)}$ in the decomposition (13). Moreover, let $\operatorname{pr}_{e}: G \rightarrow G_{e}$ be projection onto the factor $G_{e}$ in (13). If $p$ is a prime and $N$ an $\alpha$-stable closed normal subgroup 
of $G$ which is locally pro- $p$, then $\operatorname{pr}_{k}(N)=\{e\}$ for all $k \in\{1, \ldots, m\}$ such that $p_{k} \neq p$; moreover, $\operatorname{pr}_{e}(N)=\{e\}$ (see Lemma 2.11 (d) and (e)). If $p \notin\left\{p_{1}, \ldots, p_{m}\right\}$, this entails that $N=\{e\}$, whence $\{e\}$ is the largest $\alpha$ stable closed normal subgroup of $G$ which is locally pro- $p$. If $p=p_{k}$ for some $k \in\{1, \ldots, m\}$, the preceding shows that $N \subseteq G_{\left(p_{k}\right)}$, whence $G_{\left(p_{k}\right)}$ is the largest $\alpha$-stable closed normal subgroup of $G$ which is locally pro- $p$.

Remark 2.17 We mention that, in Proposition 2.16, $\left\{p_{1}, \ldots, p_{m}\right\}$ is the set of all prime divisors of $\Delta\left(\left.\alpha^{-1}\right|_{G_{\mathrm{td}}}\right)$. Since

$$
\Delta\left(\left.\alpha^{-1}\right|_{G_{\mathrm{td}}}\right)=\Delta\left(\left.\alpha^{-1}\right|_{G_{\left(p_{1}\right)}}\right) \cdots \Delta\left(\left.\alpha^{-1}\right|_{G_{\left(p_{m}\right)}}\right)
$$

this assertion will follow from Lemma 4.13.

Proposition 2.18 Let $(G, \alpha)$ be a totally disconnected locally compact contraction group which is locally pro-nilpotent and a torsion group. Let $p$ be a prime. Then $\operatorname{tor}_{p}(G)$ coincides with $G_{(p)}$, the largest closed normal subgroup of $G$ which is $\alpha$-stable and locally pro-p.

Proof. Let $p_{1}<\cdots<p_{m}$ and $S_{p}$ for $p \in\left\{p_{1}, \ldots, p_{m}\right\}$ be as in Proposition 2.16 and its proof. Then $S_{p}$ is a pro-p-group and a torsion group, whence $S_{p} \subseteq \operatorname{tor}_{p}(G)$ and hence also

$$
G_{(p)}=\bigcup_{n \in \mathbb{N}_{0}} \alpha^{-n}\left(S_{p}\right) \subseteq \operatorname{tor}_{p}(G) .
$$

Since $G=G_{\left(p_{1}\right)} \times \cdots \times G_{\left(p_{m}\right)}$, we deduce that $\operatorname{tor}_{p}(G)=\{e\}=G_{(p)}$ for all primes $p \notin\left\{p_{1}, \ldots, p_{m}\right\}$. Moreover, $\operatorname{tor}_{p}(G)=G_{(p)}$ if $p \in\left\{p_{1}, \ldots, p_{m}\right\}$.

\section{Proof of Theorem A, completed.}

To establish (c), recall from Lemma 2.6 that $H:=\operatorname{tor}(G)$ is a totally disconnected $\alpha$-stable closed subgroup of $G$. As $H$ is locally pro-nilpotent by hypothesis, Propositions 2.16 and 2.18 show that $\operatorname{tor}_{p}(H)=\operatorname{tor}_{p}(G)$ is nontrivial only for $p$ in a finite set of primes $q_{1}<\cdots<q_{m}$. Moreover, $\operatorname{tor}_{p}(H)=$ $H_{(p)}$ is locally pro- $p$ for each prime $p$ and $\operatorname{tor}(H)=\operatorname{tor}_{q_{1}}(H) \times \cdots \times \operatorname{tor}_{q_{m}}(H)$. Recalling [1.2, all assertions are established. 
Remark 2.19 We mention that the hypothesis in Theorem A(c) is also necessary for its conclusion.

To see this, let $(G, \alpha)$ be a totally disconnected, locally compact contraction group and $q_{1}<\cdots<q_{m}$ be prime numbers such that $\operatorname{tor}_{p}(G)$ is a closed subgroup of $G$ for $p \in\left\{q_{1}, \ldots, q_{m}\right\}$ and

$$
G=\operatorname{tor}_{q_{1}}(G) \times \cdots \times \operatorname{tor}_{q_{m}}(G)
$$

internally as a topological group. Then $\operatorname{tor}_{p}(G)$ is locally pro- $p$ for all $p \in$ $\left\{q_{1}, \ldots, q_{m}\right\}$ (as each compact open subgroup $U_{p}$ of $\operatorname{tor}_{p}(G)$ is both pro-finite and a $p$-group and hence a pro- $p$-group). Since $U_{q_{1}} \times \cdots \times U_{q_{m}}$ is a compact open subgroup of $G$ and pro-nilpotent, we see that $G$ is locally pro-nilpotent.

\section{Existence of equivariant global sections}

In this section, we prove Theorem C. If $(G, \alpha),(H, \beta)$, and $q$ are as in the theorem, we have decompositions $G=G_{e} \times G_{\mathrm{td}}$ and $H=H_{e} \times H_{\mathrm{td}}$ as in Theorem A. Since $q$ is a group homomorphism such that $q\left(G_{e}\right)=H_{e}$ and $q\left(G_{\mathrm{td}}\right)=H_{\mathrm{td}}($ see Lemma 2.13), we have

$$
q=\left.q\right|_{G_{e}} \times\left. q\right|_{G_{\mathrm{td}}}: G_{e} \times G_{\mathrm{td}} \rightarrow H_{e} \times H_{\mathrm{td}}
$$

If we can find a continuous section $\sigma_{1}: H_{e} \rightarrow G_{e}$ for the surjective morphism $\left.q\right|_{G_{e}}: G_{e} \rightarrow H_{e}$ of contraction groups and a continuous section $\sigma_{2}: H_{\mathrm{td}} \rightarrow G_{\mathrm{td}}$ for the surjective morphism $\left.q\right|_{G_{\mathrm{td}}}: G_{\mathrm{td}} \rightarrow H_{\mathrm{td}}$ such that $\left.\alpha\right|_{G_{e}} \circ \sigma_{1}=\left.\sigma_{1} \circ \beta\right|_{H_{e}}$ and $\left.\alpha\right|_{G_{\mathrm{td}}} \circ \sigma_{2}=\left.\sigma_{2} \circ \beta\right|_{H_{\mathrm{td}}}$, then $\sigma:=\sigma_{1} \times \sigma_{2}$ will be a continuous section for $q$ such that $\alpha \circ \sigma=\sigma \circ \beta$. Moreover, $\sigma(e)=\sigma\left(\beta^{n}(e)\right)=\alpha^{n}(\sigma(e))$ for each $n \in \mathbb{N}$ and hence $\sigma(e)=\lim _{n \rightarrow \infty} \alpha^{n}(\sigma(e))=e$. It therefore suffices to prove Theorem $\mathrm{C}$ in two cases: a) both $G$ and $H$ are totally disconnected; b) both $G$ and $H$ are connected. We start with case a); case b) will be settled afterwards, in Lemma 3.2 .

\section{Proof of Theorem $\mathrm{C}$ when $\boldsymbol{G}$ and $\boldsymbol{H}$ are totally disconnected}

Let $V \subseteq G$ be a compact open subgroup such that $\alpha(V) \subseteq V$ (see 1.8). Then $\left\{\alpha^{k}(V): k \in \mathbb{N}_{0}\right\}$ is a basis of identity neighbourhoods in $G$ (see 1.7). By 1.11, $q$ is an open map. Hence $U:=q(V)$ is a compact open subgroup of $H$. Since $\beta \circ q=q \circ \alpha$, we have $\beta(U) \subseteq U$. By 1.7. $\left\{\beta^{k}(U): k \in \mathbb{N}_{0}\right\}$ is a basis of identity neighbourhoods in $H$. Since $\beta(U)$ is open and $U$ is compact, 
the index $\ell:=[U: \beta(U)]$ is finite. We pick representatives $h_{1}, \ldots, h_{\ell}$ for the left cosets $h \beta(U) \in U / \beta(U)$ of $\beta(U)$ in $U$, such that $h_{1}=e$. We find $g_{1}, \ldots, g_{\ell} \in V$ such that $q\left(g_{j}\right)=h_{j}$ for all $j \in\{1, \ldots, \ell\}$, and $g_{1}=e$. Let $m \in \mathbb{Z}$. For $h \in \beta^{m}(U)$, we show by induction on $n \in\{m, m+1, \ldots\}$ that there are unique $j_{m}, j_{m+1}, \ldots, j_{n} \in\{1, \ldots, \ell\}$ such that

$$
h \beta^{k+1}(U)=\beta^{m}\left(h_{j_{m}}\right) \cdots \beta^{k}\left(h_{j_{k}}\right) \beta^{k+1}(U)
$$

for all $k \in\{m, \ldots, n\}$. If $n=m$, there is a unique $j_{m} \in\{1, \ldots, \ell\}$ with $h \beta^{m+1}(U)=\beta^{m}\left(h_{j_{m}}\right) \beta^{m+1}(U)$ as $\beta^{m}\left(h_{1}\right), \ldots, \beta^{m}\left(h_{\ell}\right)$ are representatives for the left cosets of $\beta^{m+1}(U)$ in $\beta^{m}(U)$. If $n>m$ and unique $j_{m}, \ldots, j_{n-1}$ have been found such that (16) holds for all $k \in\{m, \ldots, n-1\}$, then

$$
h=\beta^{m}\left(h_{j_{m}}\right) \cdots \beta^{n-1}\left(h_{j_{n-1}}\right) z
$$

for a unique $z \in \beta^{n}(U)$ and $z \beta^{n+1}(U)=\beta^{n}\left(h_{j_{n}}\right) \beta^{n+1}(U)$ for a unique $j_{n} \in$ $\{1, \ldots, \ell\}$. Then

$$
h \beta^{n+1}(U)=\beta^{m}\left(h_{j_{m}}\right) \cdots \beta^{n}\left(h_{j_{n}}\right) \beta^{n+1}(U)
$$

and $j_{n}$ is uniquely determined by this property. In fact, if (17) also holds with $i \in\{1, \ldots, \ell\}$ in place of $j_{n}$, then we can multiply both identities with $\beta^{n-1}\left(h_{j_{n-1}}\right)^{-1} \cdots \beta^{m}\left(h_{j_{m}}\right)^{-1}$ on the left. Comparing the right hand sides,

$$
\beta^{n}\left(h_{j_{n}}\right) \beta^{n+1}(U)=\beta^{n}\left(h_{i}\right) \beta^{n+1}(U)
$$

follows and hence $i=j_{n}$.

For $n \in\{m, m+1, \ldots\}$, let $f_{n}(h):=\alpha^{n}\left(g_{j_{n}}\right)$. Then $f_{n}\left(h^{\prime}\right)=f_{n}(h)$ for all $h^{\prime} \in \beta^{m}(U)$ such that $h^{\prime} \in h \beta^{n+1}(U)$, showing that the function

$$
f_{n}: \beta^{m}(U) \rightarrow \alpha^{n}(V)
$$

is locally constant and thus continuous. Define $s_{n}(h):=\alpha^{m}\left(g_{j_{m}}\right) \cdots \alpha^{n}\left(g_{j_{n}}\right)$ for $n \in\{m, m+1, \ldots\}$. By Lemma 1.15, the limit

$$
\sigma(h):=\lim _{n \rightarrow \infty} s_{n}(h)
$$

exists for all $h \in \beta^{m}(U)$, and the function $\beta^{m}(U) \rightarrow G, h \mapsto \sigma(h)$ is continuous. Given $h \in H$, there is $m \in \mathbb{Z}$ such that $h \in \beta^{m}(U)$ and our definition of $\sigma(h)$ does not depend on the choice of $m$. We therefore obtain a well-defined 
function $\sigma: H \rightarrow G, h \mapsto \sigma(h)$ which is continuous as each of the subsets $\beta^{m}(U)$ is open in $H$ and $H=\bigcup_{m \in \mathbb{Z}} \beta^{m}(U)$. Since

$$
q\left(s_{n}(h)\right)=\beta^{m}\left(q\left(g_{j_{m}}\right)\right) \cdots \beta^{n}\left(q\left(g_{j_{n}}\right)\right)=\beta^{m}\left(h_{j_{m}}\right) \cdots \beta^{n}\left(h_{j_{n}}\right) \in h \beta^{n+1}(U)
$$

for $m \in \mathbb{Z}, h \in \beta^{m}(U)$ and $n \in\{m, m+1, \ldots\}$, we see that

$$
q(\sigma(h))=\lim _{n \rightarrow \infty} q\left(s_{n}(h)\right)=h .
$$

If $h \in \beta^{m}(U)$, then $\beta(h) \in \beta^{m+1}(U)$ holds and the indices $k_{i}$ determined by $\beta(h)$ are $k_{i}=j_{i-1}$ in terms of those determined as above by $h$, for all $i \in\{m+1, m+2, \ldots\}$. Hence

$$
\begin{aligned}
\sigma(\beta(h)) & =\lim _{n \rightarrow \infty} \alpha^{m+1}\left(g_{k_{m+1}}\right) \cdots \alpha^{n}\left(g_{k_{n}}\right) \\
& =\alpha\left(\lim _{n \rightarrow \infty} \alpha^{m}\left(g_{j_{m}}\right) \cdots \alpha^{n-1}\left(g_{j_{n-1}}\right)\right)=\alpha(\sigma(h))
\end{aligned}
$$

and thus $\sigma \circ \beta=\alpha \circ \sigma$.

Lemma 3.1 Let $\mathbb{K}=\mathbb{R}$ or $\mathbb{K}$ be a field $\mathbb{Q}_{p}$ of $p$-adic numbers. Let $\mathfrak{g}$ and $\mathfrak{h}$ be finite-dimensional nilpotent Lie algebras over $\mathbb{K}$; write $*$ both for the Baker-Campbell-Hausdorff multiplication $\mathfrak{g} \times \mathfrak{g} \rightarrow \mathfrak{g}$ on $\mathfrak{g}$ and the one on $\mathfrak{h}$. If $\phi:(\mathfrak{g}, *) \rightarrow(\mathfrak{h}, *)$ is a continuous group homomorphism, then $\phi: \mathfrak{g} \rightarrow \mathfrak{h}$ is a homomorphism of Lie algebras. Thus $\operatorname{ker}(\phi)$ is an ideal of the Lie algebra $\mathfrak{g}$ and $\phi(\mathfrak{g})$ is a Lie subalgebra of $\mathfrak{h}$. Notably, $\phi(\mathfrak{g})$ is closed in $\mathfrak{h}$ and the corestriction $\left.\phi\right|^{\phi(\mathfrak{g})}: \mathfrak{g} \rightarrow \phi(\mathfrak{g})$ is an open map.

Proof. For each $x \in \mathfrak{g}$ and $n \in \mathbb{N}$, we have $\phi(n x)=\phi\left(x^{n}\right)=\phi(x)^{n}=$ $n \phi(x)$, using (8). Hence $\phi(z x)=z \phi(x)$ for all rational numbers $z$ (by unique divisibility) and thus $\phi(z x)=z \phi(x)$ for all $z \in \mathbb{K}$, by continuity of $\phi$ and density of $\mathbb{Q}$ in $\mathbb{K}$. As a consequence,

$$
L(\phi)(x)=\phi^{\prime}(0)(x)=\left.\frac{d}{d z}\right|_{z=0} \phi(z x)=\phi(x) .
$$

Thus $\phi=L(\phi)$ is a Lie algebra homomorphism. All other assertions are now immediate.

We refer to $C^{\infty}$-maps between real $C^{\infty}$-manifolds as smooth maps, as usual. If $f: M \rightarrow N$ is a mapping between such manifolds (of dimensions $m$ and $n$, respectively), we say that $f$ is differentiable at a point $x \in M$ if $\psi \circ f \circ \phi^{-1}$ is 
differentiable at $\phi(x)$ for each $C^{\infty}$-diffeomorphism $\phi$ from an open neighbourhood of $x$ in $M$ onto an open subset of $\mathbb{R}^{m}$ and each $C^{\infty}$-diffeomorphism $\psi$ from an open neighbourhood of $f(x)$ in $N$ onto an open subset of $\mathbb{R}^{n}$. By the Chain Rule, it suffices to check differentiability at $\phi(x)$ for one pair $(\phi, \psi)$.

If $(E,\|\|$.$) is a normed space over \mathbb{R}$, then we write for $x \in E$ and $r>0$

$$
B_{r}^{E}(x):=\{y \in E:\|y-x\|<r\} \text { and } \bar{B}_{r}^{E}(x):=\{y \in E:\|y-x\| \leq r\} .
$$

Lemma 3.2 Let $(G, \alpha)$ and $(H, \beta)$ be connected locally compact contraction groups and $q: G \rightarrow H$ be a surjective morphism of contraction groups. Then there exists a continuous map $\sigma: H \rightarrow G$ which is smooth on $H \backslash\{e\}$ such that $q \circ \sigma=\operatorname{id}_{H}$ and $\alpha \circ \sigma=\sigma \circ \beta$.

Proof. By Lemmas 2.3 and [3.1, we may assume that $G=(\mathfrak{g}, *)$ and $H=(\mathfrak{h}, *)$ for nilpotent Lie algebras $\mathfrak{g}$ and $\mathfrak{h}$, endowed with the BCHmultiplication; moreover, we may assume that $\alpha$ and $\beta$ are contractive Lie algebra automorphisms, and $q$ is a surjective Lie algebra homomorphism. There exists a linear map $\tau: \mathfrak{h} \rightarrow \mathfrak{g}$ such that $q \circ \tau=\operatorname{id}_{\mathfrak{h}}$. Notably, $\tau$ is a smooth section for $q$. However, $\tau$ need not be equivariant. To construct an equivariant section, we proceed as follows. By Lemma 4.9, there exists a norm $\|$.$\| on \mathfrak{h}$ with respect to which $\theta:=\|\beta\|_{\text {op }}<1$. Pick $\left.r \in\right] \theta, 1[$ and set

$$
U:=B_{1}^{\mathfrak{h}}(0), \quad W:=B_{r}^{\mathfrak{h}}(0) .
$$

Then $\bar{W}=\bar{B}_{r}^{\mathfrak{h}}(0) \subseteq U$ holds, $\bar{U}=\bar{B}_{1}^{\mathfrak{h}}(0)$ and

$$
\beta(\bar{U}) \subseteq \bar{B}_{\theta}^{\mathfrak{h}}(0) \subseteq B_{r}^{\mathfrak{h}}(0)=W .
$$

Notably, $\beta(U) \subseteq U$ and

$$
\beta^{k}(U) \subseteq W \text { for all } k \in \mathbb{N} .
$$

Moreover,

$$
U \backslash\{0\}=\bigcup_{n \in \mathbb{N}_{0}} \beta^{n}(U \backslash \beta(U))=\bigcup_{n \in \mathbb{N}_{0}} \beta^{n}(U \backslash \beta(\bar{W}))
$$

and $\mathfrak{h} \backslash\{0\}=\bigcup_{n \in \mathbb{Z}} \beta^{n}(U \backslash \beta(\bar{W}))$. For each open cover of the finitedimensional smooth manifold $\mathfrak{h}$, there exists a smooth partition of unity 
subordinate to it (cf. [10, Corollary III.2.4]). As the sets $W$ and $\mathfrak{h} \backslash \beta(\bar{U})$ form an open cover of $\mathfrak{h}$, we therefore find smooth maps $g: \mathfrak{h} \rightarrow \mathbb{R}$ and $h: \mathfrak{h} \rightarrow \mathbb{R}$ with image in $[0,1]$ such that $h+g=1$ and $\operatorname{supp}(g) \subseteq W$, $\operatorname{supp}(h) \subseteq \mathfrak{h} \backslash \beta(\bar{U})$. Note that also $\tau_{1}:=\alpha \circ \tau \circ \beta^{-1}$ is a section for $q$. We define

$$
\sigma_{0}: \mathfrak{h} \rightarrow \mathfrak{g}, \quad x \mapsto \tau(x) *\left(g(x)\left(\tau(x)^{-1} * \tau_{1}(x)\right)\right) .
$$

Then $\sigma_{0}$ is smooth and a section for $q$ (as $\operatorname{ker}(q)$ is a vector subspace of $\mathfrak{g}$ and $\left.\tau(x)^{-1} * \tau_{1}(x) \in \operatorname{ker}(q)\right)$. Since $g(x)=0$ for $x \in \mathfrak{h} \backslash W$, we have

$$
\sigma_{0}(x)=\tau(x) \text { for all } x \in \mathfrak{h} \backslash W \text {. }
$$

Since $g(x)=1$ for all $x \in \beta(\bar{U})$, we have

$$
\sigma_{0}(x)=\tau_{1}(x)=\alpha\left(\tau\left(\beta^{-1}(x)\right)\right) \text { for all } x \in \beta(\bar{U}) .
$$

We now define a map $\sigma: \mathfrak{h} \rightarrow \mathfrak{g}$ via $\sigma(0):=0$ and

$$
\sigma(x):=\alpha^{n}\left(\sigma_{0}\left(\beta^{-n}(x)\right)\right) \text { if } n \in \mathbb{Z} \text { and } x \in \beta^{n}(U \backslash \beta(\bar{W})) \text {. }
$$

To see that $\sigma$ is well-defined, let $x \in \mathfrak{h}$ and $n<m$ be integers such that

$$
x \in \beta^{n}(U \backslash \beta(\bar{W})) \cap \beta^{m}(U \backslash \beta(\bar{W})) .
$$

Then $U \backslash \beta(\bar{W}) \cap \beta^{m-n}(U \backslash \beta(\bar{W})) \neq \emptyset$, whence $\beta^{m-n}(U)$ cannot be a subset of $\beta(W)$ and thus $m-n-1 \leq 0$, by (18). Hence $m=n+1$. Now $y:=$ $\beta^{-n-1}(x)=\beta^{-m}(x) \in U \backslash \beta(\bar{W})$ and

$$
\beta(y) \in(U \backslash \beta(\bar{W})) \cap \underbrace{\beta(U \backslash \beta(\bar{W}))}_{\subseteq \beta(U) \subseteq U} \subseteq \beta(U) \backslash \beta(\bar{W})=\beta(U \backslash \bar{W}),
$$

whence

$$
y \in U \backslash \bar{W}
$$

Then

$$
\begin{aligned}
\alpha^{n}\left(\sigma_{0}\left(\beta^{-n}(x)\right)\right) & =\alpha^{n}\left(\sigma_{0}(\beta(y))\right)=\alpha^{n+1}(\tau(y))=\alpha^{n+1}\left(\sigma_{0}(y)\right) \\
& =\alpha^{n+1}\left(\sigma_{0}\left(\beta^{-n-1}(x)\right)\right)=\alpha^{m}\left(\sigma_{0}\left(\alpha^{-m}(x)\right)\right) .
\end{aligned}
$$

In fact, the second equality holds by (21), applied to $\beta(y)$ in place of $x$, which is possible by (22). The third equality holds by (20), which is applicable 
by (23). In view of (24), $\sigma$ is well-defined.

By definition, $\sigma$ coincides with the smooth section $\alpha^{n} \circ \sigma_{0} \circ \beta^{-n}$ of $q$ on the open set $\beta^{n}(U \backslash \beta(\bar{W}))$ for each $n \in \mathbb{Z}$. As the latter sets cover $\mathfrak{h} \backslash\{0\}$ (cf. (19)), we see that $\left.\sigma\right|_{\mathfrak{h} \backslash\{0\}}$ is smooth and a section for $q$. Thus $\sigma$ is a section for $q$, since also $q(\sigma(0))=q(0)=0$.

To see that $\sigma$ is continuous, it only remains to check its continuity at 0 . To this end, let $V \subseteq \mathfrak{g}$ be a 0 -neighbourhood. Since $\sigma_{0}(\bar{U})$ is compact and $\alpha$ is contractive, there exists $n_{0} \in \mathbb{N}_{0}$ such that

$$
\alpha^{n}\left(\sigma_{0}(\bar{U})\right) \subseteq V \text { for all } n \geq n_{0} .
$$

As a consequence, $\sigma(x) \in V$ for all $x \in \bigcup_{n \geq n_{0}} \beta^{n}(U \backslash \beta(\bar{W}))=\beta^{n}(U) \backslash\{0\}$ (cf. (19)). Since $\sigma(0)=0 \in V$, we deduce that $\sigma\left(\beta^{n}(U)\right) \subseteq V$. Thus $\sigma$ is continuous at 0 .

The condition $\alpha(\sigma(x))=\sigma(\beta(x))$ is trivial if $x=0$. If $x \in \mathfrak{h} \backslash\{0\}$, then $x \in \beta^{n}(U \backslash \beta(\bar{W}))$ for some $n \in \mathbb{Z}$ (cf. (19) ). Then $\beta(x) \in \beta^{n+1}(U \backslash \beta(\bar{W}))$ and we get

$$
\sigma(\beta(x))=\alpha^{n+1}\left(\sigma_{0}\left(\beta^{-n-1}(\beta(x))\right)\right)=\alpha\left(\alpha^{n}\left(\sigma_{0}\left(\beta^{-n}(x)\right)\right)\right)=\alpha(\sigma(x)),
$$

using the piecewise definition of $\sigma$ for the first equality and the last.

Let us briefly discuss differentiability of equivariant sections at $e$.

Lemma 3.3 In the setting of Lemma 3.2, the following are equivalent:

(a) $\sigma$ can be chosen as a smooth map;

(b) $\sigma$ can be chosen differentiable at e;

(c) There exists a linear map $\tau: L(H) \rightarrow L(G)$ such that $L(q) \circ \tau=\mathrm{id}_{L(H)}$ and $L(\alpha) \circ \tau=\tau \circ L(\beta)$.

Proof. The implication "(a) $\Rightarrow(\mathrm{b})$ " is trivial and also "(c) $\Rightarrow(\mathrm{a})$ ", once we identify $(G, H, q, \alpha, \beta)$ with $(L(G), L(H), L(q), L(\alpha), L(\beta))$ as explained in the preceding proof. To see that (b) implies (c), consider a continuous section $\tau: L(H) \rightarrow L(G)$ for $L(q)$ which is differentiable at 0 and satisfies

$$
L(\alpha) \circ \tau=\tau \circ L(\beta) .
$$


Then $L(q) \circ \tau=\operatorname{id}_{L(H)}$ has the derivative

$$
\operatorname{id}_{L(H)}=L(q) \circ \tau^{\prime}(0)
$$

at 0 , by the Chain Rule. Thus $\tau^{\prime}(0): L(H) \rightarrow L(G)$ is a linear section for $L(q)$. Differentiating (25) at 0 , we get $L(\alpha) \circ \tau^{\prime}(0)=\tau^{\prime}(0) \circ L(\beta)$.

We mention that $\sigma$ in Lemma 3.2 cannot always be chosen as a smooth map.

Example 3.4 Recall from the introduction that $\Omega_{\infty}$ is the set of monic irreducible real polynomials $f$ such that all eigenvalues of $f$ have absolute value less than 1 , and that $\alpha_{f^{n}}$ is the automorphism of $E_{f^{n}}=\mathbb{R}[X] / f^{n} \mathbb{R}[X]$ induced by multiplication by $X$, for $n \in \mathbb{N}$. For $f \in \Omega_{\infty}$, the mapping $q: E_{f^{2}} \rightarrow E_{f}, g+f^{2} \mathbb{R}[X] \mapsto g+f \mathbb{R}[X]$ is a surjective morphism of contraction groups from $\left(E_{f^{2}}, \alpha_{f^{2}}\right)$ to $\left(E_{f}, \alpha_{f}\right)$ which does not admit an equivariant linear section (as the latter would be an $\mathbb{R}[X]$-module homomorphism and thus $E_{f^{2}} \cong\left(E_{f}\right)^{2}$, which is absurd). By Lemma 3.3, $q$ cannot admit a smooth equivariant section.

\section{More on homomorphisms, series and simple factors}

In this section, we first provide further results concerning continuous group homomorphisms between locally compact contraction groups, including a proof of the statements of Theorem G. These results ensure a certain "closed product property", which is essential for our proof of a Jordan-Hölder Theorem. After a brief review of facts concerning contractive automorphisms of real vector spaces, we then establish the classification described in Theorem F. The section closes with preparatory results for Section 5 .

\section{Results related to Theorem G}

Proposition 4.1 Let $\phi: G \rightarrow H$ be a continuous group homomorphism between contractible locally compact groups $G$ and $H$. Then $\phi(G)$ is closed in $H$ if and only if $\phi(\operatorname{tor}(G))$ is closed. In this case, $\left.\phi\right|^{\phi(G)}: G \rightarrow \phi(G)$ is an open map.

Proof. Let $\alpha$ and $\beta$ be contractive automorphisms for $G$ and $H$, respectively. Recall from Theorem A that $\operatorname{tor}(G)$ is a closed subgroup of $G$ and $G=$ 
$G_{e} \times G_{p_{1}} \times \cdots \times G_{p_{n}} \times \operatorname{tor}(G)$ for certain primes $p_{1}<\cdots<p_{n}$ and $p$-adic Lie groups $G_{p}$ which are $\alpha$-stable closed normal subgroups of $G$. Set $G_{p}:=\{e\}$ for primes $p \notin\left\{p_{1}, \ldots, p_{n}\right\}$. Likewise, $\operatorname{tor}(H)$ is closed in $H$ and

$$
H=H_{e} \times H_{q_{1}} \times \cdots \times H_{q_{m}} \times \operatorname{tor}(H)
$$

for certain primes $q_{1}<\cdots<q_{m}$ and $p$-adic Lie groups $H_{p}$ which are $\beta$-stable closed normal subgroups of $H$. By Proposition 2.14, we have $\phi(\operatorname{tor}(G)) \subseteq$ tor $(H)$ and

$$
\phi(G)=\phi\left(G_{e}\right) \times \phi\left(G_{q_{1}}\right) \times \cdots \times \phi\left(G_{q_{m}}\right) \times \phi(\operatorname{tor}(G)),
$$

where $\phi\left(G_{e}\right)$ is closed in $H_{e}$ and $\phi\left(G_{p}\right)$ is closed in $H_{p}$ for all $p \in\left\{q_{1}, \ldots, q_{m}\right\}$, by Lemmas 2.3, 2.4 and 3.1. In view of (26), the desired equivalence follows. Now $G$ is $\sigma$-compact, whence also $\phi(G)$ is $\sigma$-compact. If $\phi(G)$ is closed and hence locally compact, the Open Mapping Theorem [8, Theorem 5.29] shows that $\left.\phi\right|^{\phi(G)}$ is an open map.

For example, $\phi(G)$ is closed for each continuous group homomorphism $\phi: G \rightarrow H$ between contractible locally compact groups such that $\operatorname{tor}(G) \subseteq$ $\operatorname{ker}(\phi)$ (which is the case if $G$ or $H$ is torsion-free).

The next example shows that $\phi(G)$ need not be closed in Proposition 4.1.

Example 4.2 Consider the additive group $A:=\mathbb{F}_{p}((t))=\mathbb{F}_{p}^{(-\mathbb{N})} \times \mathbb{F}^{\mathbb{N}_{0}}$ with the right shift $\alpha: A \rightarrow A, f \mapsto t f$. Then the map

$$
\phi: A \rightarrow A, \quad\left(a_{n}\right)_{n \in \mathbb{Z}} \mapsto \sum_{n=1}^{\infty} a_{-2 n} t^{-n}+\sum_{n=0}^{\infty} a_{-2 n-1} t^{n}
$$

is a continuous group homomorphism. The image of $\phi$ is the set $\mathbb{F}_{p}^{(\mathbb{Z})}$ of all finitely supported sequences in $A$. Thus $\phi(A)$ is a dense proper subgroup of $A$, and so $\phi(A)$ is not closed in $A$. The co-restriction $\left.\phi\right|^{\phi(A)}: A \rightarrow \phi(A)$ is not an open map, as otherwise $\phi(A)$ would be isomorphic to the locally compact group $A / \operatorname{ker}(\phi)$, whence $\phi(A)$ would be complete and hence closed in $A$ (a contradiction).

Corollary 4.3 If $\phi:(G, \alpha) \rightarrow(H, \alpha)$ is a morphism of locally compact contraction groups, then $\phi(G)$ is a closed subgroup of $H$ and $\left.\phi\right|^{\phi(G)}$ is an open map. 
Proof. We know from Lemma 2.6 that tor $(G)$ is an $\alpha$-stable closed subgroup of $G$ and totally disconnected. Likewise, $\operatorname{tor}(H)$ is a closed subgroup of $H$ and totally disconnected. Hence $\phi(\operatorname{tor}(G))$ is closed in $\operatorname{tor}(H)$ and hence in $H$, by 1.11. The assertions now follow from Proposition 4.1.

In particular, locally compact contraction groups always have the following "closed product propertiy", which previously had only been recorded for totally disconnected $G$ (see [7, Corollary 3.2]).

Corollary 4.4 Let $(G, \alpha)$ be a locally compact contraction group, $N$ be an $\alpha$-stable closed subgroup of $G$ and $H$ be an $\alpha$-stable closed subgroup of $G$ which normalizes $N$. Then $N H$ is a closed $\alpha$-stable subgroup of $G$.

Proof. Let $H$ act on $N$ via $h . n:=h n h^{-1}$ for $h \in H$ and $n \in N$. The corresponding semidirect product $N \rtimes H$ is locally compact group and the map $\left.\alpha\right|_{N} \times\left.\alpha\right|_{H}$ a contractive automorphism thereof. As the product map

$$
\pi: N \rtimes H \rightarrow G, \quad(n, h) \mapsto n h
$$

is a morphism of contraction groups, we deduce with Corollary 4.3 that $N H=\pi(N \rtimes H)$ is closed in $G$.

\section{Composition series and the Jordan-Hölder Theorem}

4.5 A series (51) of contraction groups is called a refinement of a series

$$
\{e\}=H_{0} \triangleleft H_{1} \triangleleft \cdots \triangleleft H_{m}=G
$$

of contraction groups if $\left\{H_{0}, \ldots, H_{m}\right\} \subseteq\left\{G_{0}, \ldots, G_{n}\right\}$. If $m=n$ and there is a permutation $\pi$ of $\{1, \ldots, n\}$ such that

$$
G_{j} / G_{j-1} \cong H_{\pi(j)} / H_{\pi(j)-1}
$$

as contraction groups for all $j \in\{1, \ldots, n\}$, then (5) and (27) are called isomorphic series of contraction groups.

Lemma 4.6 Let $(G, \alpha)$ be a locally compact contraction group. If $G$ is connected, then $n \leq \operatorname{dim}_{\mathbb{R}} L(G)$ for each series $\{e\}=G_{0} \triangleleft G_{1} \triangleleft \cdots \triangleleft G_{n}=G$ of contraction groups for $(G, \alpha)$ such that $G_{j-1}$ is a proper subgroup of $G_{j}$ for all $n \in\{1, \ldots, n\}$. In particular, $(G, \alpha)$ admits a composition series of contraction groups. 
Proof. We may assume that $G=(\mathfrak{g}, *)$ for a nilpotent real Lie algebra $\mathfrak{g}$ and that $\alpha$ is a contractive Lie algebra automorphism. Lemma 3.1 implies that $G_{j}$ is a Lie subalgebra of $\mathfrak{g}$ for each $j \in\{1, \ldots, n\}$, and $\operatorname{dim}_{\mathbb{R}}\left(G_{j-1}\right)<\operatorname{dim}_{\mathbb{R}}\left(G_{j}\right)$. The first assertion follows from this and also the second, as each properly ascending series (5) with maximal $n$ will be a composition series.

The following result generalizes the case of totally disconnected groups discussed in [7, Theorem 3.3].

Theorem 4.7 For each locally compact contraction group $(G, \alpha)$, we have:

(a) There exists a composition series $\{e\}=G_{0} \triangleleft G_{1} \triangleleft \cdots \triangleleft G_{n}=G$ of contraction groups for $(G, \alpha)$.

(b) Zassenhaus Lemma: Any two series of contraction groups for $(G, \alpha)$ admit refinements which are isomorphic as series of contraction groups.

(c) Jordan-Hölder Theorem: Every series of contraction groups for $(G, \alpha)$ can be refined to a composition series of contraction groups. Moreover, any two composition series of contraction groups for $(G, \alpha)$ are isomorphic as series of contraction groups.

Proof. (a) By [15, Proposition 4.2], $G=G_{e} \times G_{\mathrm{td}}$ internally for a totally disconnected, $\alpha$-stable closed normal subgroup $G_{\mathrm{td}}$ of $G$. By Lemma 4.6, $G_{e}$ has a composition series $\{e\}=G_{0} \triangleleft G_{1} \triangleleft \cdots \triangleleft G_{n}=G_{e}$ of contraction groups. By [7, Theorem 3.3], $G_{\mathrm{td}}$ has a composition series $\{e\}=H_{0} \triangleleft H_{1} \cdots \triangleleft H_{m}=$ $G_{\mathrm{td}}$ of contraction groups. Then

$$
\{e\}=G_{0} \triangleleft G_{1} \triangleleft \cdots \triangleleft G_{n}=G_{e} \times H_{0} \triangleleft G_{e} \times H_{1} \triangleleft \cdots \triangleleft G_{e} \times H_{m}=G
$$

is a composition series of contraction groups for $(G, \alpha)$.

(b) and (c) holds by [7, Proposition 2.4], in view of (a) and the "closed product property" established in Corollary 4.4 .

\section{Contractive automorphisms of real and complex vector spaces}

We briefly review some facts concerning contractive automorphisms of real (or complex) finite-dimensional vector spaces.

Lemma 4.8 Let $\alpha: E \rightarrow E$ be a $\mathbb{C}$-linear automorphism of a finite-dimensional complex vector space $E$. Then the following conditions are equivalent: 
(a) $\alpha$ is contractive;

(b) $|\lambda|<1$ for all eigenvalues $\lambda$ of $\alpha$ in $\mathbb{C}$;

(c) There exists a norm $\|$.$\| on E such that \alpha$ has operator norm $\|\alpha\|_{\mathrm{op}}<1$ with respect to $\|$.$\| .$

Proof. The implication "(c) $\Rightarrow(\mathrm{a})$ " is trivial and also "(a) $\Rightarrow(\mathrm{b})$ " (since $\neg(\mathrm{b}) \Rightarrow \neg(\mathrm{a}))$. To complete the proof, it suffices to prove the implication "(b) $\Rightarrow(\mathrm{c})$ " in the case when matrix describing $\alpha$ with respect to a suitable basis of $E$ consists of a single Jordan block (using the convention that ones appear below the diagonal) 4 So, let $\lambda \in \mathbb{C}, k \in \mathbb{N}$ and $0 \neq v \in E$ such that $\left(\alpha-\lambda \mathrm{id}_{E}\right)^{k}(v)=0$ and the vectors $v_{j}:=\left(\alpha-\lambda \mathrm{id}_{E}\right)^{j}(v)$ form a basis for $E$ for $j \in\{0,1, \ldots, k-1\}$. For $\theta>0$, also the vectors $\theta^{-j} v_{j}$ form a basis for $E$ for $j \in\{0,1, \ldots, k-1\}$. Since

$$
\alpha\left(\sum_{j=0}^{k-1} x_{j} \theta^{-j} v_{j}\right)=\lambda x_{0} \theta^{0} v_{0}+\sum_{j=1}^{k-1}\left(\lambda x_{j}+\theta x_{j-1}\right) \theta^{-j} v_{j}
$$

for $x_{0}, \ldots, x_{k-1} \in \mathbb{C}$, we have $\|\alpha\|_{\text {op }} \leq|\lambda|+\theta$ with respect to the norm on $E$ given by $\left\|\sum_{j=0}^{k-1} x_{j} \theta^{-j} v_{j}\right\|:=\max \left\{\left|x_{0}\right|, \ldots,\left|x_{k-1}\right|\right\}$. Thus $\|\alpha\|_{\text {op }}<1$ if we choose $\theta \in] 0,1-|\lambda|[$.

Lemma 4.9 Let $\alpha: E \rightarrow E$ be an $\mathbb{R}$-linear automorphism of a finite-dimensional real vector space $E$. The following conditions are equivalent:

(a) $\alpha$ is contractive;

(b) The corresponding complex linear map $\alpha_{\mathbb{C}}: E_{\mathbb{C}} \rightarrow E_{\mathbb{C}}$ is contractive, where $E_{\mathbb{C}}:=E \otimes_{\mathbb{R}} \mathbb{C}$ and $\alpha_{\mathbb{C}}:=\alpha \otimes \mathrm{id}_{\mathbb{C}} ;$

(c) There exists a norm $\|$. $\|$ on $E$ such that $\alpha$ has operator norm $\|\alpha\|_{\mathrm{op}}<1$ with respect to $\|$.$\| .$

\footnotetext{
${ }^{4}$ In general, $E$ is a direct sum $\bigoplus_{j=1}^{n} E_{j}$ of $\alpha$-stable vector subspaces $E_{j}$ with this property. Then $\|\alpha\|_{\text {op }}<1$ with respect to the norm $\|$.$\| on E$ given by $\left\|x_{1}+\cdots+x_{n}\right\|:=$ $\max \left\{\left\|x_{1}\right\|_{1}, \ldots,\left\|x_{n}\right\|_{n}\right\}$ for $x_{j} \in E_{j}$, where $\|\cdot\|_{j}$ is a norm on $E_{j}$ with respect to which $\left\|\left.\alpha\right|_{E_{j}}\right\|_{\text {op }}<1$.
} 
Proof. The implication " $(\mathrm{c}) \Rightarrow(\mathrm{a})$ " is trivial.

$(\mathrm{a}) \Rightarrow(\mathrm{b})$ : Considering $E_{\mathbb{C}}$ as a real vector space, we have $E_{\mathbb{C}}=E \oplus i E \cong$ $E \times E$ and $\alpha_{\mathbb{C}}$ corresponds to $\alpha \times \alpha$, which is contractive.

$(\mathrm{b}) \Rightarrow(c)$ : If (b) holds, then there exists a norm $\|$.$\| on E_{\mathbb{C}}$ with respect to which $\left\|\alpha_{\mathbb{C}}\right\|_{\text {op }}<1$. Identify $x \in E$ with $x \otimes 1 \in E_{\mathbb{C}}$. Then $\|\alpha\|_{\text {op }}<1$ with respect to the restriction of $\|\cdot\|$ to $E$.

\section{Classification of the simple locally compact contraction groups}

A classification of the simple totally disconnected contraction groups was obtained in [7, Theorem A and Proposition 6.3]. The following theorem completes the picture for general locally compact contraction groups. Recall from the introduction that $\Omega_{\infty}$ is the set of all monic irreducible polynomials $f$ over $\mathbb{R}$ such that $|\lambda|<1$ for all zeros $\lambda$ of $f$ in $\mathbb{C}$. Given a polynomial $f \in \Omega_{\infty}$, we endow $E_{f}:=\mathbb{R}[X] / f \mathbb{R}[X]$ with the $\mathbb{R}$-linear automorphism $\alpha_{f}$ given by multiplication of representatives by $X$.

We assume that the reader is familiar with the theory of finitely generated modules over a principal ideal domain (like $\mathbb{R}[X]$ ) and its relations to normal forms for endomorphisms of finite-dimensional vector spaces (see, e.g., [9, Chapter 3]). We now establish the classification described in Theorem F.

Proof of Theorem F. As $G=G_{e} \times G_{\mathrm{td}}$ internally and $(G, \alpha)$ is simple, we must have $G_{e}=\{e\}$ or $G_{\mathrm{td}}=\{e\}$, establishing the first assertion. If $G$ is connected, then $G$ is a nilpotent real Lie group (see Lemma 2.3), whence its centre $Z(G)$ is a non-trivial $\alpha$-stable closed normal subgroup of $G$ and thus $G=Z(G)$, as $(G, \alpha)$ is assumed simple. Hence $G$ is abelian. As a consequence, the Lie algebra $L(G)$ is abelian, i.e., $[x, y]=0$ for all $x, y \in L(G)$ (cf. Proposition 7 in [2, Chapter III, §9.3]). The BCH-multiplication on $L(G)$ therefore is the addition map,

$$
x * y=x+y \text { for all } x, y \in L(G) .
$$

Thus $(G, \alpha)$ is isomorphic to $((L(G),+), L(\alpha))$ as a contraction group (see Lemma 2.3). We may hence assume that $G$ is a finite-dimensional real vector space $E$ and $\alpha: E \rightarrow E$ a contractive linear automorphism. If $p \in \mathbb{R}[X]$ is the characteristic polynomial of $\alpha$, then $|\lambda|<1$ for all roots of $p$ in $\mathbb{C}$, by Lemmas 4.9 and 4.8. Consider $E$ as an $\mathbb{R}[X]$-module via $X . v=\alpha(v)$ for $v \in E$. Since $(E, \alpha)$ is a simple contraction group, $p$ coincides with the minimal polynomial of $\alpha$ and $\mathbb{R}[X] / p \mathbb{R}[X] \cong E$ is a simple $\mathbb{R}[X]$-module, 
whence $p$ is irreducible. Thus $p \in \Omega_{\infty}$ and $(E, \alpha) \cong \mathbb{R}[X] / p \mathbb{R}[X]=\left(E_{p}, \alpha_{p}\right)$.

Conversely, $\left(E_{f}, \alpha_{f}\right)$ is a contraction group for each $f \in \Omega_{\infty}$, by Lemmas 4.9 and 4.8. Since each closed $\alpha$-stable subgroup of $E_{f}$ is a vector subspace (cf. Lemmas 2.3 and 3.1), the contraction group $\left(E_{f}, \alpha_{f}\right)$ is simple as $E_{f}=$ $\mathbb{R}[X] / f \mathbb{R}[X]$ is a simple $\mathbb{R}[X]$-module (since $f$ is irreducible). If $f, g \in \Omega_{\infty}$ and $\phi:\left(E_{f}, \alpha_{f}\right) \rightarrow\left(E_{g}, \alpha_{g}\right)$ is an isomorphism of contraction groups, then $\phi$ is linear (cf. Lemmas 2.3 and 3.1 ) and hence an isomorphism of $\mathbb{R}[X]$-modules. From

$$
\mathbb{R}[X] / f \mathbb{R}[X]=E_{f} \cong E_{g}=\mathbb{R}[X] / g \mathbb{R}[X]
$$

we deduce that $f=g$.

\section{The composition length}

Definition 4.10 Let $(G, \alpha)$ be a locally compact contraction group. Theorem 4.7 (c) shows that each composition series $\{e\}=G_{0} \triangleleft G_{1} \triangleleft \cdots \triangleleft G_{n}=G$ of contraction groups for $(G, \alpha)$ has the same length $n \in \mathbb{N}_{0}$. We call $n$ the composition length of $(G, \alpha)$ and write $\ell(G):=n$.

Two simple observations will be useful later.

Lemma 4.11 If $(G, \alpha)$ is a locally compact contraction group and $N \subseteq G$ an $\alpha$-stable closed normal subgroup, then

$$
\ell(G)=\ell(N)+\ell(G / N)
$$

if we endow $N$ with the contractive automorphism $\left.\alpha\right|_{N}$ and $G / N$ with $\bar{\alpha}: x N \mapsto \alpha(x) N$.

Proof. Let $q: G \rightarrow G / N$ be the canonical quotient map. Then a composition series for $\left(N,\left.\alpha\right|_{N}\right)$ and the preimages in $G$ under $q$ of a composition series for $(G / N, \bar{\alpha})$ combine to a composition series for $(G, \alpha)$.

Lemma 4.12 If $\phi: G \rightarrow H$ is a morphism between locally compact contraction groups $(G, \alpha)$ and $(H, \beta)$, then

$$
\ell(G)=\ell(\operatorname{ker} \phi)+\ell(\phi(G)) .
$$

Proof. Since $\phi(G) \cong G / \operatorname{ker} \phi$, this follows from Lemma 4.11. 


\section{Composition length in locally pro-p groups}

Lemma 4.13 Let $(G, \alpha)$ be a locally compact contraction group such that $G$ is locally pro-p. Then the following holds:

(a) If $G \neq\{e\}$, then $\Delta\left(\alpha^{-1}\right)=p^{k}$ for some $k \in \mathbb{N}$.

(b) If $G$ is a torsion group, then $\Delta\left(\alpha^{-1}\right)=p^{\ell(G)}$, where $\ell(G)$ is the composition length of $(G, \alpha)$. Moreover, the order of each group element of $G$ divides $p^{\ell(G)}$.

Proof. (a) Let $V \subseteq G$ be a compact open subgroup such that $\alpha(V) \subseteq V$ (see 1.8). Since $\bigcap_{n \in \mathbb{N}_{0}} \alpha^{n}(V)=\{e\}$ as a consequence of 1.7, $\alpha(V)$ is a proper subgroup of $V$. Since $V$ is a pro-p-group (see Lemma 2.8) and $\alpha(V)$ is open in $V$, we deduce that $\Delta\left(\alpha^{-1}\right)=[V: \alpha(V)]=p^{k}$ for some $k \in \mathbb{N}$ (see (41)).

(b) If $H:=\mathbb{F}_{p}((t))$ and $\beta(z):=t z$ for $z \in H$, then $U:=\mathbb{F}_{p} \llbracket t \rrbracket$ is a compact open subgroup of $H$ and $\beta(U)=t \mathbb{F}_{p} \llbracket t \rrbracket$ is a subgroup of index $p$ in $U$. By (44),

$$
\Delta\left(\beta^{-1}\right)=[U: \beta(U)]=p .
$$

If $(G, \alpha)$ has a composition series (5), give $G_{j} / G_{j-1}$ the contractive automorphism $\alpha_{j}$ taking $x G_{j-1}$ to $\alpha(x) G_{j-1}$. Since $G_{j} / G_{j-1}$ is a simple contraction group, locally pro- $p$, and a torsion group, the classification in [7, Theorem A] shows that $\left(G_{j} / G_{j-1}, \alpha_{j}\right) \cong(H, \beta)$ with $H=\mathbb{F}_{p}((t))$ as above. Thus

$$
\Delta\left(\alpha^{-1}\right)=\Delta\left(\left(\alpha_{1}\right)^{-1}\right) \cdots \Delta\left(\left(\alpha_{n}\right)^{-1}\right)=\Delta\left(\beta^{-1}\right)^{n}=p^{n}=p^{\ell(G)}
$$

indeed (cf. [5, Proposition III.13.20]). As each subquotient $G_{j} / G_{j-1}$ is a torsion group of finite exponent $p$, we deduce from (5) that $G$ is a torsion group of exponent dividing $p^{n}=p^{\ell(G)}$.

\section{Abelian contraction groups}

In this section, we prove the structure theorems concerning locally compact abelian contraction groups described in the introduction (Theorems D and E). In particular, we classify those locally compact contraction groups which are abelian torsion groups (Corollary [5.6). Up to isomorphism, there are only countably many. By contrast, for each $p$ one easily finds uncountable sets of contraction groups which are abelian $p$-adic Lie groups and pairwise non-isomorphic, and likewise for contraction groups which are abelian real Lie groups (see Example 5.2). 
5.1 Every locally compact contraction group $(G, \alpha)$ such that $G$ is an abelian $p$-adic Lie group has an abelian $p$-adic Lie algebra $L(G)$, whence the BCHmultiplication coincides with addition and $(G, \alpha)$ is isomorphic to $(L(G),+)$ with the contractive linear automorphism $L(\alpha)$ (as a special case of 2.4). Likewise, every abelian connected locally compact contraction group $(G, \alpha)$ is a real Lie group whose Lie algebra $L(G)$ is abelian, and $(G, \alpha)$ is isomorphic to $(L(G),+)$ endowed with the contractive linear automorphism $L(\alpha)$ (cf. 2.3) .

Example 5.2 For $a \in \mathbb{Q}_{p}$ of $p$-adic absolute value $|a|_{p}<1$, consider the multiplication operator $\mu_{a}: \mathbb{Q}_{p} \rightarrow \mathbb{Q}_{p}, z \mapsto a z$. Then $\left(\mathbb{Q}_{p}, \mu_{a}\right)$ is an abelian contraction group. If also $b \in \mathbb{Q}_{p}$ with $|b|_{p}<1$ and $\phi:\left(\mathbb{Q}_{p}, \mu_{a}\right) \rightarrow\left(\mathbb{Q}_{p}, \mu_{b}\right)$ is an isomorphism of contraction groups, then the homomorphism $\phi$ is $\mathbb{Q}_{p^{-}}$ linear, by Lemma 3.1. Hence

$$
a \phi(1)=\phi(a 1)=\left(\phi \circ \mu_{a}\right)(1)=\left(\mu_{b} \circ \phi\right)(1)=b \phi(1),
$$

entailing that $a=b$ and thus $\left(\mathbb{Q}_{p}, \mu_{a}\right)=\left(\mathbb{Q}_{p}, \mu_{b}\right)$. Analogous arguments apply if $\mathbb{Q}_{p}$ is replaced with $\mathbb{R}$.

For each $m \in \mathbb{N}$ and prime number $p$, we write $F_{p^{m}}:=\mathbb{Z} / p^{m} \mathbb{Z}$ and endow $F_{p^{m}}((t))$ with the contractive automorphism $\alpha: z \mapsto t z$.

Remark 5.3 (a) Note that $\left(F_{p^{m}}((t)), \alpha\right)$ has composition length $m$ since

$$
\{0\}=p^{m} F_{p^{m}}((t)) \triangleleft p^{m-1} F_{p^{m}}((t)) \triangleleft \cdots \triangleleft p^{0} F_{p^{m}}((t))=F_{p^{m}}((t))
$$

is a composition series of $\alpha$-stable closed normal subgroups.

[In fact, $p^{k}\left(F_{p^{m}}((t))\right)$ is the set of all $x=\sum_{i=i_{0}}^{\infty} x_{i} t^{i}$ in $F_{p^{m}}((t))$ such that $i_{0} \in \mathbb{Z}$ and $x_{i} \in p^{k} F_{p^{m}}$ for all $i \geq i_{0}$. As a consequence, $p^{k-1} F_{p^{m}}((t)) / p^{k} F_{p^{m}}((t)) \cong$ $\mathbb{F}_{p}((t))$ for each $k \in\{1, \ldots, m\}$.]

(b) Let $x=\sum_{i=i_{0}}^{\infty} x_{i} t^{i} \in F_{p^{m}}((t))$ with $i_{0} \in \mathbb{Z}$ and $x_{i} \in F_{p^{m}}$ for all $i \geq i_{0}$. Let $k \in\{0,1, \ldots, m\}$. Then $p^{k} x=0$ if and only if $p^{k} x_{i}=0$ for all $i \geq i_{0}$, which holds if and only if $x_{i} \in p^{m-k} F_{p^{m}}$. Thus

$$
\left\{x \in F_{p^{m}}((t)): p^{k} x=0\right\}=p^{m-k} F_{p^{m}}((t)) .
$$

Lemma 5.4 Let $(G, \alpha)$ be a totally disconnected, locally compact contraction group such that $G$ is abelian. If $x \in G$ is an element of prime power order $p^{m}$ with $m \in \mathbb{N}$, then there is a unique morphism of contraction groups

$$
F_{p^{m}}((t)) \rightarrow G \text { taking } t^{0} \text { to } x \text {. }
$$


Its image $\langle x\rangle_{\alpha}$ is the smallest $\alpha$-stable closed subgroup of $G$ containing $x$, and its co-restriction

$$
\theta_{x}: F_{p^{m}}((t)) \rightarrow\langle x\rangle_{\alpha}
$$

is an isomorphism of contraction groups if we use $\left.\alpha\right|_{\langle x\rangle_{\alpha}}$ on the right hand side. Notably, $\langle x\rangle_{\alpha} \cong F_{p^{m}}((t))$.

Proof. Let + be the group operation on $G$. Let $U \subseteq G$ be a compact open subgroup such that $\alpha(U) \subseteq U$ (see 1.8). Since $\alpha$ is contractive, we have $\alpha^{k}(x) \in U$ for some $k \in \mathbb{N}_{0}$. After replacing $U$ with $\alpha^{-k}(U)$, we may assume that $x \in U$. Then

$$
U \supseteq \alpha(U) \supseteq \alpha^{2}(U) \supseteq \cdots
$$

is a basis of identity neighbourhoods of $G$ consisting of compact open subgroups (cf. 1.7). We claim that a) the limit

$$
\phi_{x}(z):=\sum_{j=N}^{\infty} k_{j} \alpha^{j}(x)
$$

exists in $G$ for all $N \in \mathbb{Z}$ and all

$$
z=\sum_{j=N}^{\infty} k_{j} t^{j} \in F_{p^{m}}((t))
$$

with $k_{j} \in\left\{0,1, \ldots, p^{m}-1\right\}$; and b) the function $\phi_{x}: F_{p^{m}}((t)) \rightarrow G, z \mapsto \phi_{x}(z)$ is continuous and a homomorphism. As the sets $V_{N}$ of all $z$ as in (32) form an open cover of $F_{p^{m}}((t))$ for $N \in \mathbb{Z}$ consisting of nested compact open subgroups, it suffices to fix $N \in \mathbb{Z}$ and show c) that the above limit exists for $z \in V_{N}$; and d) that $\left.\phi_{x}\right|_{V_{N}}$ is a continuous homomorphism. Note that Lemma 1.15] can be applied using the continuous functions

$$
f_{n}: V_{N} \rightarrow \alpha^{N+n}(U) \subseteq G, \quad \sum_{j=N}^{\infty} k_{j} t^{j} \mapsto k_{N+n} \alpha^{N+n}(x) .
$$

Hence c) holds and $\left.\phi_{x}\right|_{V_{N}}$ is continuous. Being a pointwise limit of group homomorphisms, also $\left.\phi_{x}\right|_{V_{N}}$ is a homomorphism. By construction, we have

$$
\phi_{x}(t z)=\alpha\left(\phi_{x}(z)\right) \text { for all } z \in F_{p^{m}}((t)) .
$$

Thus $\phi_{x}$ is a morphism of contraction groups, entailing that its image $\langle x\rangle_{\alpha}$ is an $\alpha$-stable closed subgroup of $G$. Since the set $F_{p^{m}}^{(\mathbb{Z})}$ of finitely supported 
sequences is dense in $F_{p^{m}}((t))$, the elements $\alpha^{n}(x)$ with $n \in \mathbb{Z}$ generate a dense subgroup of $\langle x\rangle_{\alpha}$. As a consequence, $\langle x\rangle_{\alpha}$ is the smallest $\alpha$-stable closed subgroup of $G$ which contains $x$. To complete the proof, note that

$$
m \leq \ell\left(\langle x\rangle_{\alpha}\right) \leq \ell\left(F_{p^{m}}((t))\right)=m
$$

by Lemma 4.13(b), 4.12, and Remark 5.3. Thus $\ell\left(\langle x\rangle_{\alpha}\right)=\ell\left(F_{p^{m}}((t))\right)$, whence $\ell\left(\operatorname{ker} \theta_{x}\right)=0$ (by 4.12) and thus $\operatorname{ker} \theta_{x}=\{0\}$. By 1.11, $\theta_{x}$ is an isomorphism of topological groups.

Lemma 5.5 For each prime $p$ and $m \in \mathbb{N}_{0}$, the lattice of $\alpha$-stable closed subgroups of $F_{p^{m}}((t))$ is totally ordered; it has the form

$$
\{0\}=p^{m} F_{p^{m}}((t))<p^{m-1} F_{p^{m}}((t))<\cdots<p^{0} F_{p^{m}}((t))=F_{p^{m}}((t)) .
$$

If $k \in\{0,1, \ldots, m\}$ and $x \in F_{p^{m}}((t))$ is an element of order $p^{k}$, then $\langle x\rangle_{\alpha}=$ $p^{m-k} F_{p^{m}}((t))$.

Proof. Let $H$ be an $\alpha$-stable closed subgroup of $F_{p^{m}}((t))$ and $k \in\{0, \ldots, m\}$ be minimal with $p^{k} H=\{0\}$. Let $x \in H$ be an element of order $p^{k}$. Then

$$
\langle x\rangle_{\alpha} \leq H \leq p^{m-k} F_{p^{m}}((t)),
$$

using Remark 5.3(b). As $\langle x\rangle_{\alpha} \cong F_{p^{k}}((t))$ (see Lemma 5.4) and $p^{m-k} F_{p^{m}}((t)) \cong$ $F_{p^{k}}((t))$ both have composition length $k$ (see Remark 5.3(a)), 4.11 shows that

$$
p^{m-k} F_{p^{m}}((t)) /\langle x\rangle_{\alpha}=\{0\}
$$

as it has composition length 0 . Thus $\langle x\rangle_{\alpha}=H=p^{m-k} F_{p^{m}}((t))$.

Proof of Theorem D: Existence. By Theorem A(c),

$$
G=\operatorname{tor}_{p_{1}}(G) \times \cdots \times \operatorname{tor}_{p_{m}}(G)
$$

internally as a contraction group for certain primes $p_{1}<\cdots<p_{m}$, such that $\operatorname{tor}_{p}(G) \neq\{e\}$ for all $p \in\left\{p_{1}, \ldots, p_{m}\right\}$. If we can show that $\operatorname{tor}_{p_{k}}(G)$ is isomorphic to $A_{k}^{(-\mathbb{N})} \times A_{k}^{\mathbb{N}_{0}}$ with the right shift for a finite abelian group $A_{k}$, then $G$ is isomorphic to $F^{(-\mathbb{N})} \times F^{\mathbb{N}_{0}}$ with the right shift for the finite abelian group $F:=A_{1} \times \cdots \times A_{m}$. After replacing $G$ with $\operatorname{tor}_{p}(G)$ for $p \in\left\{p_{1}, \ldots, p_{m}\right\}$, we may therefore assume now that $G$ is a non-trivial $p$-group and locally pro- $p$ 
for some prime $p$. We show by induction on $\ell(G) \in \mathbb{N}$ that $G \cong F^{(-\mathbb{N})} \times F^{\mathbb{N}}$ for an abelian group $F$ of order $|F|=p^{\ell(G)}$. If $\ell(G)=1$, then $(G, \alpha)$ is a simple totally disconnected contraction group which is a torsion group, abelian and locally pro- $p$, whence $(G, \alpha)$ is isomorphic to $\mathbb{F}_{p}^{(-\mathbb{N})} \times \mathbb{F}_{p}^{\mathbb{N}_{0}}$ by the classification in [7, Theorem A], and the assertion holds. If $\ell(G)>1$, pick an element $x \in G$ of maximal order $p^{k}$. Then

$$
\langle x\rangle_{\alpha} \cong F_{p^{k}}((t)) \cong F_{p^{k}}^{(-\mathbb{N})} \times F_{p^{k}}^{\mathbb{N}_{0}}
$$

by Lemma 5.4, and $k \leq \ell(G)$ by 4.11 and Remark 5.3(a). Let $q: G \rightarrow G /\langle x\rangle_{\alpha}$ be the canonical quotient morphism and endow $G /\langle x\rangle_{\alpha}$ with the contractive automorphism $\bar{\alpha}$ determined by $\bar{\alpha}(q(y))=q(\alpha(y))$ for $y \in G$. If $k=\ell(G)$, then $\langle x\rangle_{\alpha}=G$ is a shift on a restricted power of $F_{p^{k}}$, where $\left|F_{p^{k}}\right|=p^{k}=p^{\ell(G)}$. If $k<\ell(G)$, then $G /\langle x\rangle_{\alpha}$ has composition length $\ell(G)-k$ (see 4.11), whence

$$
G /\langle x\rangle_{\alpha} \cong A^{(-\mathbb{N})} \times A^{\mathbb{N}_{0}}
$$

with the right shift for an abelian group $A$ of order $|A|=p^{\ell(G)-k}$, by the inductive hypothesis. The Structure Theorem for Finite Abelian Groups (cf. [11, 4.2.6] or [12, Corollary 10.22]) shows that

$$
A \cong F_{p^{k_{1}}} \times \cdots \times F_{p^{k_{n}}}
$$

for some $n \in \mathbb{N}$ and $k_{1}, \ldots, k_{n} \in \mathbb{N}$ such that $k_{1}+\cdots+k_{n}=\ell(G)-k$. Thus

$$
A^{(-\mathbb{N})} \times A^{\mathbb{N}_{0}} \cong \bigoplus_{j=1}^{n} F_{p^{k_{j}}}((t)) .
$$

As a consequence, there exist $y_{1}, \ldots, y_{n} \in G /\langle x\rangle_{\alpha}$ such that

$$
G /\langle x\rangle_{\alpha}=\left\langle y_{1}\right\rangle_{\bar{\alpha}} \times \cdots \times\left\langle y_{n}\right\rangle_{\bar{\alpha}}
$$

internally and $F_{p^{k_{j}}}((t)) \cong\left\langle y_{j}\right\rangle_{\bar{\alpha}}$ for all $j \in\{1, \ldots, n\}$, by means of isomorphisms $\theta_{y_{j}}$ taking $t^{0}$ to $y_{j}$, as described in Lemma 5.4.

We claim that, for each $j \in\{1, \ldots, n\}$, there exists $x_{j} \in G$ such that $q\left(x_{j}\right)=y_{j}$ and $x_{j}$ has order $p^{k_{j}}$. If this is true, then

$$
\sigma_{j}:=\theta_{x_{j}} \circ \theta_{y_{j}}^{-1}:\left\langle y_{j}\right\rangle_{\bar{\alpha}} \rightarrow\left\langle x_{j}\right\rangle_{\alpha}
$$

is a morphism of contraction groups for $j \in\{1, \ldots, n\}$, whence also

$$
\sigma: G /\langle x\rangle_{\alpha} \rightarrow G, \quad g_{1}+\cdots+g_{n} \mapsto \sigma_{1}\left(g_{1}\right)+\cdots+\sigma_{n}\left(g_{n}\right)
$$


for $\left(g_{1}, \ldots, g_{n}\right) \in\left\langle y_{1}\right\rangle_{\bar{\alpha}} \times \cdots \times\left\langle y_{n}\right\rangle_{\bar{\alpha}}$ is a morphism of contraction groups. Then $q \circ \sigma$ is a morphism of contraction groups which takes $y_{j}$ to itself and hence coincides with the identity map on $\left\langle y_{j}\right\rangle_{\bar{\alpha}}$, for each $j \in\{1, \ldots, n\}$. Thus

$$
q \circ \sigma=\mathrm{id}
$$

on $G /\langle x\rangle_{\alpha}$, entailing that $G=\operatorname{im}(\sigma) \times \operatorname{ker}(q)=\left\langle x_{1}\right\rangle_{\alpha} \times \cdots \times\left\langle x_{n}\right\rangle_{\alpha} \times\langle x\rangle_{\alpha}$ internally as a topological group (and a contraction group). Thus $G \cong$ $F^{(-\mathbb{N})} \times F^{\mathbb{N}_{0}}$ with $F:=A \times F_{p_{k}}$.

To prove the claim, pick $z_{j} \in G$ such that $q\left(z_{j}\right)=y_{j}$. Then $z_{j}$ has order $p^{\ell_{j}}$ with $k_{j} \leq \ell_{j} \leq k$, and $p^{k_{j}} z_{j} \in\langle x\rangle_{\alpha}$ is an element of order $p^{\ell_{j}-k_{j}}$ and hence contained in

$$
p^{k-\ell_{j}+k_{j}}\langle x\rangle_{\alpha}
$$

by Lemma 5.5. Thus $p^{k_{j}} z_{j}=p^{k-\ell_{j}+k_{j}} r_{j}$ for some $r_{j} \in\langle x\rangle_{\alpha}$ and thus $p^{k_{j}} z_{j}=$ $p^{k_{j}}\left(p^{k-\ell_{j}} r_{j}\right)$ with $k-\ell_{j} \geq 0$. Then $x_{j}:=z_{j}-p^{k-\ell_{j}} r_{j}$ is an element such that $q\left(x_{j}\right)=q\left(z_{j}\right)=y_{j}$ (whence its order is at least $p^{k_{j}}$ ) and $p^{k_{j}} x_{j}=0$, whence $x_{j}$ has order $p^{k_{j}}$.

The uniqueness assertion will be proved at the end of this section.

Proof of Theorem E. Existence: By Theorem A, there are prime numbers $p_{1}<\cdots<p_{n}$ and $p$-adic Lie groups $G_{p}$ for $p \in\left\{p_{1}, \ldots, p_{n}\right\}$ which are $\alpha$-stable closed subgroups of $G$ such that

$$
G=G_{e} \times G_{p_{1}} \times \cdots \times G_{p_{n}} \times \operatorname{tor}(G)
$$

moreover, $\operatorname{tor}(G) \cong F^{(-\mathbb{N})} \times F^{\mathbb{N}_{0}}$ for a finite abelian group $F$, by Theorem $\mathrm{D}$. Abbreviate $G_{\infty}:=G_{e}$. If $\beta$ is an endomorphism of a finite-dimensional vector space $E$ over a field $\mathbb{K}$, let us write $(E, \beta)$ for $E$, endowed with the $\mathbb{K}[X]$-module structure determined by $X . v=\beta(v)$ for $v \in E$. Let $p \in$ $\left\{p_{1}, \ldots, p_{n}, \infty\right\}$. In view of 5.1 , the Structure Theorem for Finitely Generated Modules over Principal Ideal Domains shows that

$$
\left(G_{p},\left.\alpha\right|_{G_{p}}\right) \cong \bigoplus_{f} \bigoplus_{n \in \mathbb{N}}\left(E_{f^{n}}, \alpha_{f^{n}}\right)^{\mu(p, f, n)},
$$

where $f$ ranges through the set of all monic irreducible polynomials with coefficients in $\mathbb{Q}_{p}$ and $\mu(p, f, n) \neq 0$ for only finitely many $(f, n)$. As each $\alpha_{f^{n}}$ with $\mu(p, f, n)>0$ is contractive (like $\left.\alpha\right|_{G_{p}}$ ), we must have $f \in \Omega_{p}$ for such $(p, f, n)$ (see Lemmas 4.9 and 4.8). We may therefore assume that $f \in \Omega_{p}$ 
in (33). For each prime $p$ such that $p \notin\left\{p_{1}, \ldots, p_{n}\right\}$, we set $\mu(p, f, n):=0$ for all $f \in \Omega_{p}$ and $n \in \mathbb{N}$. Finally, by the Structure Theorem for Finite Abelian Groups (cf. [11, 4.2.6] or [12, Corollary 10.22]),

$$
F \cong \bigoplus_{p \in \mathbb{P}} \bigoplus_{n \in \mathbb{N}}\left(F_{p^{n}}\right)^{\nu(p, n)}
$$

where $\nu(p, n) \in \mathbb{N}_{0}$ is non-zero for only finitely many $(p, n)$. Hence

$$
F^{(-\mathbb{N})} \times F^{\mathbb{N}_{0}} \cong \bigoplus_{p \in \mathbb{P}} \bigoplus_{n \in \mathbb{N}}\left(F_{p^{n}}((t))\right)^{\nu(p, n)},
$$

showing that $(G, \alpha)$ is isomorphic to a direct sum of the form $(*)$ asserted in Theorem E. Conversely, every group of the form $(*)$ described in Theorem $\mathrm{E}$ is a locally compact contraction group (using Lemmas 4.9, 4.8, and [17. Lemma 3.4 and its proof]).

Uniqueness: If $(*)$ holds, then $\left(G_{e},\left.\alpha\right|_{G_{e}}\right)$ is isomorphic to the connected component $\bigoplus_{f \in \Omega_{\infty}} \bigoplus_{n \in \mathbb{N}}\left(E_{f^{n}}, \alpha_{f^{n}}\right)^{\mu(\infty, f, n)}$ and uniquely determines the integers $\mu(\infty, f, n)$ by uniqueness of the $\mu(\infty, f, n)$ in (33) . For each prime $p$ such that $p \notin\left\{p_{1}, \ldots, p_{n}\right\}$, we must have $\mu(p, f, n)=0$ for all $f \in \Omega_{p}$ and $n \in \mathbb{N}$, by uniqueness in Theorem $\mathrm{A}(\mathrm{b})$. For $p \in\left\{p_{1}, \ldots, p_{n}\right\}$, uniqueness in Theorem A(b) implies that the contraction group $\left(G_{p},\left.\alpha\right|_{G_{p}}\right)$ is isomorphic to $\bigoplus_{f \in \Omega_{p}} \bigoplus_{n \in \mathbb{N}}\left(E_{f^{n}}, \alpha_{f^{n}}\right)^{\mu(p, f, n)}$ and uniquely determines the $\mu(p, f, n)$ by uniqueness of the $\mu(p, f, n)$ in (33). Finally, if $(*)$ holds then

$$
\left(\operatorname{tor}_{p}(G),\left.\alpha\right|_{\operatorname{tor}_{p}(G)}\right) \cong \bigoplus_{n \in \mathbb{N}}\left(F_{p^{n}}((t)), \alpha_{p^{n}}\right)^{\nu(p, n)}
$$

for each prime number $p$. We may therefore assume that $G=\operatorname{tor}_{p}(G)$ to establish uniqueness of the $\nu(p, n)$ for $n \in \mathbb{N}$ and a given prime number $p$. For $m \in \mathbb{N}$, let $T_{m}$ be the $\alpha$-stable closed subgroup of $G$ consisting of elements $x \in G$ whose order divides $p^{m}$. Then $T_{m} / T_{m-1}$ is trivial if $m$ is greater than the largest $n$ such that $\nu(p, n) \neq 0$ and is isomorphic to $\left(F_{p}((t)), \alpha_{p}\right)^{\nu(p, n)}$ if $m$ equals this value. This isomorphism determines $\nu(p, n)$ uniquely in this case. For smaller values of $m$, we have

$$
S_{m} / S_{m-1} \cong \bigoplus_{n \geq m}\left(F_{p}((t)), \alpha_{p}\right)^{\nu(p, n)},
$$

and $\nu(p, m)$ may thus be determined once $\nu(p, n)$ is known for all $n>m$.

The following is immediate from Theorem E and the preceding discussion. 
Corollary 5.6 Endowed with the right shift $\alpha_{p^{n}}$ in each component,

$$
\bigoplus_{p \in \mathbb{P}} \bigoplus_{n \in \mathbb{N}}\left(\mathbb{F}_{p^{n}}((t)), \alpha_{p^{n}}\right)^{\nu(p, n)}
$$

is a locally compact abelian torsion contraction group for each family

$$
(\nu(p, n))_{(p, n) \in \mathbb{P} \times \mathbb{N}} \in \mathbb{N}_{0}^{(\mathbb{P} \times \mathbb{N})} .
$$

Any locally compact abelian torsion contraction group is isomorphic to a contraction group of the preceding form, for a uniquely determined family

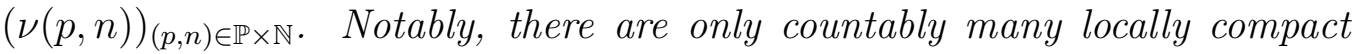
abelian contraction groups which are torsion groups, up to isomorphism.

Proof of Theorem D, completed: Uniqueness. Assume that $(G, \alpha)$ is isomorphic to $F^{(-\mathbb{N})} \times F^{\mathbb{N}_{0}}$ with the right shift for a finite abelian group $F$. By the Structure Theorem for Finite Abelian Groups,

$$
F \cong \bigoplus_{p \in \mathbb{P}} \bigoplus_{n \in \mathbb{N}}\left(F_{p^{n}}\right)^{\nu(p, n)}
$$

for $\nu(p, n) \in \mathbb{N}_{0}$ which are non-zero for only finitely many $(p, n)$. Then

$$
(G, \alpha) \cong \bigoplus_{p \in \mathbb{P}} \bigoplus_{n \in \mathbb{N}}\left(F_{p^{n}}((t))\right)^{\nu(p, n)}
$$

as a contraction group. By Theorem $\mathrm{E}$, the $\nu(p, n)$ in (36) are uniquely determined by $(G, \alpha)$. Hence $F$ is determined up to isomorphism.

\section{Two auxiliary results concerning extensions}

We record two observations concerning extensions, for later use.

Lemma 6.1 Let $\left(A, \alpha_{A}\right),(\widehat{G}, \widehat{\alpha})$ and $(G, \alpha)$ be totally disconnected, locally compact contraction groups. Let

$$
\{e\} \rightarrow A \stackrel{\iota}{\rightarrow} \widehat{G} \stackrel{q}{\rightarrow} G \rightarrow\{e\}
$$

be an extension of contraction groups such that $\left(A, \alpha_{A}\right)$ and $(G, \alpha)$ are simple contraction groups and both $A$ and $G$ are abelian. If $\widehat{G}$ has non-trivial centre, then $\widehat{G}$ is abelian or $Z(\widehat{G})=\iota(A)$ and $G$ is 2-step nilpotent. 
Proof. After replacing $A$ with $\iota(A)$, we may assume that $A \subseteq \widehat{G}$ and $\iota$ is the inclusion map. Since $Z(\widehat{G})$ is a closed $\widehat{\alpha}$-stable normal subgroup of $\widehat{G}$ and $q: \widehat{G} \rightarrow G$ is a surjective morphism of contraction groups, the image $q(Z(\widehat{G}))$ is a closed $\alpha$-stable normal subgroup of $G$ (see 1.11). Hence $q(Z(\widehat{G}))=\{e\}$ or $q(Z(\widehat{G}))=G$. In the first case, $Z(\widehat{G})$ is a non-trivial, $\alpha_{A}$-stable closed normal subgroup of $A$, whence $Z(\widehat{G})=A$ by simplicity of $\left(A, \alpha_{A}\right)$; as a consequence, $\widehat{G}$ is 2-step nilpotent. In the second case, $\widehat{G}=Z(\widehat{G}) A$ is abelian.

In the next lemma, we use notation as in Appendix A. Note that, in the situation of A.1, the trivial homomorphism $\gamma: G \rightarrow \operatorname{Aut}(A), x \mapsto \operatorname{id}_{A}$ always satisfies (65).

Lemma 6.2 Let $(G, \alpha)$ and $\left(A, \alpha_{A}\right)$ be totally disconnected, locally compact contraction groups with $A$ abelian. Let $\omega_{1}, \omega_{2} \in Z_{\mathrm{eq}}^{2}(G, A)$ with respect to the trivial homomorphism $\gamma: G \rightarrow \operatorname{Aut}(A), x \mapsto \mathrm{id}_{A}$ and

$$
\phi: A \times_{\omega_{1}} G \rightarrow A \times_{\omega_{2}} G
$$

be an isomorphism of contraction groups such that $\phi(A \times\{e\})=A \times\{e\}$. Let $\phi_{A}$ be the automorphism of the contraction group $A$ determined by $\phi(x, e)=$ $\left(\phi_{A}(x), e\right)$ for $x \in A$ and $\bar{\phi}$ be the automorphism of the contraction group $G$ determined by $\bar{\phi}\left(\operatorname{pr}_{2}(x, y)\right)=\operatorname{pr}_{2}(\phi(x, y))$ for $x \in A$ and $y \in G$. Then there exists $\beta \in B_{\mathrm{eq}}^{2}(G, A)$ such that

$$
\omega_{2}=\phi_{A} \circ \omega_{1} \circ\left(\bar{\phi}^{-1} \times \bar{\phi}^{-1}\right)+\beta
$$

using additive notation for $A$.

Proof. It is immediate that

$$
\omega_{0}:=\phi_{A} \circ \omega_{1} \circ\left(\bar{\phi}^{-1} \times \bar{\phi}^{-1}\right) \in Z_{\mathrm{eq}}^{2}(G, A)
$$

and a straightforward calculation shows that the equivariant homeomorphism

$$
\phi_{A}^{-1} \times \bar{\phi}^{-1}: A \times_{\omega_{0}} G \rightarrow A \times_{\omega_{1}} G,(x, y) \mapsto\left(\phi_{A}^{-1}(x), \bar{\phi}^{-1}(y)\right)
$$

is a group homomorphism (and thus an isomorphism of contraction groups). Hence also

$$
\psi:=\phi \circ\left(\phi_{A}^{-1} \times \bar{\phi}^{-1}\right): A \times_{\omega_{0}} G \rightarrow A \times_{\omega_{2}} G
$$


is an isomorphism of contraction groups. By construction, we have

$$
\psi(x, e)=\phi\left(\phi_{A}^{-1}(x), e\right)=\left(\phi_{A}\left(\phi_{A}^{-1}(x)\right), e\right)=(x, e)
$$

for all $x \in A$ and thus $\psi \circ \iota=\iota$ with $\iota: A \rightarrow A \times G, x \mapsto(x, e)$. Moreover, $\mathrm{pr}_{2} \circ \psi=\mathrm{pr}_{2}$ since $\mathrm{pr}_{2} \circ \phi=\bar{\phi} \circ \mathrm{pr}_{2}$. Hence $\psi$ is an isomorphism between the extensions

$$
\{e\} \rightarrow A \stackrel{\iota}{\rightarrow} A \times \times_{\omega_{0}} G \stackrel{\mathrm{pr}_{2}}{\rightarrow}\{e\} \text { and }\{e\} \rightarrow A \stackrel{\iota}{\rightarrow} A \times_{\omega_{2}} G \stackrel{\mathrm{pr}_{2}}{\rightarrow}\{e\}
$$

of totally disconnected, locally compact contraction groups. Therefore $\beta:=\omega_{2}-\omega_{0} \in B_{\text {eq }}^{2}(G, A)$ (see Proposition A.11).

\section{Description of biadditive cocycles}

Let $p$ be a prime, $A:=\mathbb{F}_{p}((t))$ and $\alpha: A \rightarrow A$ be the contractive automorphism $f \mapsto t f$. We let $A$ act on itself via $\gamma: A \rightarrow \operatorname{Aut}(A), x \mapsto \operatorname{id}_{A}$, i.e., we consider central extensions of $A$ by $A$. Thus, a continuous equivariant 2-cocycle $\omega: A \times A \rightarrow A$ is a continuous map satisfying the cocycle identity

$$
\omega(y, z)-\omega(x+y, z)+\omega(x, y+z)-\omega(x, y)=0 \text { for all } x, y, z \in A
$$

and the equivariance condition $\alpha \circ \omega=\omega \circ(\alpha \times \alpha)$, i.e.,

$$
t \omega(x, y)=\omega(t x, t y) \text { for all } x, y \in A \text {. }
$$

As a consequence,

$$
t^{k} \omega(x, y)=\omega\left(t^{k} x, t^{k} y\right) \text { for all } x, y \in A \text { and } k \in \mathbb{Z} .
$$

The cocycle condition (37) is satisfied in particular if a map $\omega: A \times A \rightarrow A$ is biadditive, i.e., $\omega(x+y, z)=\omega(x, z)+\omega(y, z)$ for all $x, y, z \in A$ and $\omega(x, y+z)=\omega(x, y)+\omega(x, z)$. Then $\omega(x, 0)=\omega(0, x)=0$ for all $x \in A$.

If $\omega: A \times A \rightarrow A$ is a continuous, equivariant, biadditive map, we define

$$
b_{m}(\omega):=\omega\left(t^{0}, t^{m}\right) \text { for } m \in \mathbb{Z} .
$$

Then

$$
b_{m}(\omega)=\omega\left(t^{0}, t^{m}\right) \rightarrow \omega\left(t^{0}, 0\right)=0
$$


as $m \rightarrow \infty$ and

$$
t^{m} b_{-m}(\omega)=t^{m} \omega\left(t^{0}, t^{-m}\right)=\omega\left(t^{m}, t^{0}\right) \rightarrow 0
$$

as $m \rightarrow \infty$. The two-sided sequences $\left(b_{m}(\omega)\right)_{m \in \mathbb{Z}}$ are therefore elements of the set $B$ of all $\left(a_{m}\right)_{m \in \mathbb{Z}}$ with $a_{m} \in A$ such that

$$
a_{m} \rightarrow 0 \text { and } t^{m} a_{-m} \rightarrow 0 \text { as } m \rightarrow \infty .
$$

Let $Z_{\text {bil }}^{2}(A, A) \subseteq Z_{\text {eq }}^{2}(A, A)$ be the set of all continuous, equivariant, biadditive (and hence $\mathbb{F}_{p}$-bilinear) maps $\omega: A \times A \rightarrow A$. For example, for each $n \in \mathbb{Z}$ we have $\omega_{n} \in Z_{\text {bil }}^{2}(A, A)$ if we define

$$
\omega_{n}(x, y):=\sum_{i=i_{0}}^{\infty} x_{i} y_{i+n} t^{i}
$$

for all $x=\sum_{i=i_{0}}^{\infty} x_{i} t^{i}, y=\sum_{j=j_{0}}^{\infty} y_{j} t^{j}$ with $i_{0}, j_{0} \in \mathbb{Z}$ and $x_{i}, y_{j} \in \mathbb{F}_{p}$ for integers $i \geq i_{0}$ and $j \geq j_{0}$ (and $y_{j}:=0$ for integers $j<j_{0}$ here and in the following). Note that the limit in (41) exists and defines a continuous $A$-valued function on each ball

$$
\bar{B}_{p^{-i_{0}}}^{A}(0):=\left\{x \in A:|x| \leq p^{-i_{0}}\right\}
$$

(and hence on $A$ ) as

$$
f_{i}: \bar{B}_{p^{-i_{0}}}^{A}(0) \times A \rightarrow t^{i} \mathbb{F}_{p} \llbracket t \rrbracket, \quad(x, y) \mapsto x_{i} y_{i+n} t^{i}
$$

is a continuous mapping for each integer $i \geq i_{0}$, and Lemma 1.15 applies.

We mention that

$$
\omega_{n}\left(t^{i_{0}}, t^{j_{0}}\right)=\sum_{i=i_{0}}^{\infty} \delta_{i_{0}, i} \delta_{j_{0}, i+n} t^{i}=\delta_{j_{0}, i_{0}+n} t^{i_{0}} \text { for all } i_{0}, j_{0} \in \mathbb{Z}
$$

(using Kronecker's delta, considered as an element of $\mathbb{F}_{p} \subseteq \mathbb{F}_{p}((t))$ ). Notably,

$$
\omega_{n}\left(t^{0}, t^{j}\right)=\delta_{n, j} \text { for all } j, n \in \mathbb{Z} .
$$

Observe that

$$
\left|\omega_{n}(x, y)\right| \leq|x| \text { for all } n \in \mathbb{Z} \text { and } x, y \in A .
$$

This is clear if $x=0$, as $\omega_{n}(x, y)=0$ then. If $x \neq 0$, write $x=\sum_{i=i_{0}}^{\infty} x_{i} t^{i}$ with $x_{i_{0}} \neq 0$ and $y=\sum_{j=j_{0}} y_{j} t^{j}$. By (41),,$\left|\omega_{n}(x, y)\right| \leq p^{-i_{0}}=|x|$. 
Proposition 7.1 The mapping $b: Z_{\mathrm{bil}}^{2}(A, A) \rightarrow B, \omega \mapsto\left(b_{m}(\omega)\right)_{m \in \mathbb{Z}}$ is a bijection.

Proof. The domain and range of $b$ are groups under pointwise addition and $b$ is a homomorphism. If $b(\omega)=0$, then $0=b_{m}(\omega)=\omega\left(t^{0}, t^{m}\right)$ for each $m \in \mathbb{Z}$ and hence also $\omega\left(t^{n}, t^{m}\right)=t^{n} \omega\left(t^{0}, t^{m-n}\right)=t^{n} b_{m-n}(\omega)=0$. Since $\omega$ is continuous biadditive and $\left\{t^{n}: n \in \mathbb{Z}\right\}$ generates a dense subgroup of $A$, we deduce that $\omega=0$. Thus $\operatorname{ker}(b)=\{0\}$ and hence $b$ is injective. To see that $b$ is surjective, let $a=\left(a_{m}\right)_{m \in \mathbb{Z}} \in B$. We claim that both series needed to define

$$
\omega_{a}(x, y):=\sum_{n=0}^{\infty} a_{n} \omega_{n}(x, y)+\sum_{n=1}^{\infty} a_{-n} \omega_{-n}(x, y)
$$

are convergent and define continuous $A$-valued functions of $(x, y) \in A \times A$. Then $\omega_{a}$ is biadditive and equivariant, as these properties are inherited by $\mathbb{F}_{p}((t))$-linear combinations of biadditive equivariant maps and pointwise limits of such. Hence $\omega_{a} \in Z_{\text {bil }}^{2}(A, A)$. As $\omega_{n}\left(t^{0}, t^{m}\right)=\delta_{n, m}$ by (43), we have

$$
\omega_{a}\left(t^{0}, t^{m}\right)=\sum_{n=0}^{\infty} a_{n} \omega_{n}\left(t^{0}, t^{m}\right)+\sum_{n=1}^{\infty} a_{-n} \omega_{-n}\left(t^{0}, t^{m}\right)=a_{m}
$$

for all $m \in \mathbb{Z}$ and thus $b\left(\omega_{a}\right)=a$, whence $b$ is surjective and hence bijective. To prove the claim, let $i_{0} \in \mathbb{Z}$. The sequence $\left(\rho_{n}\right)_{n \in \mathbb{N}_{0}}$ with

$$
\rho_{n}:=\sup \left\{\left|a_{k}\right| p^{-i_{0}}: k \geq n\right\}
$$

is monotonically decreasing and $\lim _{n \rightarrow \infty} \rho_{n}=0$. Let $x:=\sum_{i=i_{0}}^{\infty} x_{i} t^{i}$ and $y:=\sum_{i=i_{0}}^{\infty} y_{i} t^{i}$ with $x_{i}, y_{i} \in \mathbb{F}_{p}$ for $i \geq i_{0}$. For each $k \in \mathbb{N}_{0}$, we have

$$
\left|a_{k} \omega_{k}(x, y)\right|=\left|a_{k}\right|\left|\sum_{i=i_{0}}^{\infty} x_{i} y_{i+k} t^{i}\right| \leq\left|a_{k}\right|\left|t^{i_{0}}\right|=\left|a_{k}\right| p^{-i_{0}} \leq \rho_{k}
$$

and thus $a_{k} \omega_{k}(x, y) \in \bar{B}_{\rho_{k}}^{A}(0)$. Using Lemma 1.15, we deduce that the first sum in (45) converges to a continuous function of $(x, y) \in \bar{B}_{p^{-i_{0}}}^{A}(0) \times \bar{B}_{p^{-i_{0}}}^{A}(0)$.

Also $\tau_{n}:=\sup \left\{\left|t^{k} a_{-k}\right| p^{-i_{0}}: k \geq n\right\}$ defines a monotonically decreasing sequence $\left(\tau_{n}\right)_{n \in \mathbb{N}}$ such that $\lim _{n \rightarrow \infty} \tau_{n}=0$. For $k \in \mathbb{N}$ we have

$$
\begin{aligned}
\left|a_{-k} \omega_{-k}(x, y)\right| & =\left|a_{-k}\right|\left|\sum_{i=i_{0}+k}^{\infty} x_{i} y_{i-k} t^{i}\right|=\left|a_{-k}\right|\left|t^{k}\right|\left|\sum_{j=i_{0}}^{\infty} x_{j+k} y_{j} t^{j}\right| \\
& \leq\left|a_{-k} t^{k}\right|\left|t^{i_{0}}\right|=\left|a_{-k} t^{k}\right| p^{-i_{0}} \leq \tau_{k} .
\end{aligned}
$$


Using Lemma 1.15, we find that $\sum_{k=1}^{\infty} a_{-k} \omega_{-k}(x, y)$ converges for all $(x, y) \in$ $\bar{B}_{p^{-i_{0}}}^{A}(0) \times \bar{B}_{p^{-i_{0}}}^{A}(0)$ and defines a continuous $A$-valued function thereof.

\section{An uncountable set of contraction groups}

For $A$ as in Section 7 and $s=\left(s_{n}\right)_{n \in \mathbb{N}} \in\{0,1\}^{\mathbb{N}}$, we define a function $\eta_{s}: A \times A \rightarrow A$ via

$$
\eta_{s}(x, y):=\sum_{n=1}^{\infty} s_{n} t^{n} \omega_{2 n}(x, y) \text { for } x, y \in A,
$$

with $\omega_{n}$ as in (41). Thus $\eta_{s}=\omega_{a}$ (as in (45)) with $a_{2 n}:=s_{n} t^{n}$ for all $n \in \mathbb{N}$, $a_{n}:=0$ for $n \in-\mathbb{N}_{0}$ or $n \in 2 \mathbb{N}-1$. As a consequence, $\eta_{s} \in Z_{\text {bil }}^{2}(A, A)$. Note that $\{0,1\}^{\mathbb{N}}$ is an uncountable set of continuum cardinality. We show:

Theorem 8.1 The contraction groups $\left(A \times \eta_{s} A, \alpha \times \alpha\right)$ for $s \in\{0,1\}^{\mathbb{N}}$ are pairwise not isomorphic. Thus, if $r, s \in\{0,1\}^{\mathbb{N}}$ and there exists an isomorphism $\psi: A \times_{\eta_{s}} A \rightarrow A \times_{\eta_{r}} A$ of topological groups such that $(\alpha \times \alpha) \circ \psi=$ $\psi \circ(\alpha \times \alpha)$, then $r=s$. In particular, the map

$$
\{0,1\}^{\mathbb{N}} \rightarrow H_{\mathrm{eq}}^{2}(A, A), \quad s \mapsto\left[\eta_{s}\right]
$$

is injective.

Several lemmas will help us to establish the theorem. Given $a \in \mathbb{F}_{p}((t))^{\times}$, let $\mu_{a}: \mathbb{F}_{p}((t)) \rightarrow \mathbb{F}_{p}((t))$ be the multiplication operator $x \mapsto x a$. Since $\mu_{a}(t x)=$ txa $=t \mu_{a}(x)$ for all $x \in A$, we see that $\mu_{a}$ is an element of the group $\operatorname{Aut}(A, \alpha)$ of all automorphisms of the contraction group $(A, \alpha)$.

Lemma 8.2 The map $\varepsilon$ : $\operatorname{Aut}(A, \alpha) \rightarrow \mathbb{F}_{p}((t))^{\times}, \phi \mapsto \phi(1)$ is an isomorphism of groups with inverse $a \mapsto \mu_{a}$.

Proof. Since $\mu_{a}(1)=a$ for each $a \in \mathbb{F}_{p}((t))$, the map $\varepsilon$ is surjective. If $\phi \in$ $\operatorname{Aut}(A, \alpha)$ and $a:=\phi(1)$, then $\phi\left(t^{i}\right)=\left(\phi \circ \alpha^{i}\right)(1)=\left(\alpha^{i} \circ \phi\right)(1)=t^{i} a=\mu_{a}\left(t^{i}\right)$ for each $i \in \mathbb{Z}$. As a consequence, for each $x \in A$, say $x=\sum_{i=i_{0}}^{\infty} x_{i} t^{i}$ with $i_{0} \in \mathbb{Z}$ and $x_{i} \in \mathbb{F}_{p}$, we have

$$
\phi(x)=\sum_{i=i_{0}}^{\infty} x_{i} \phi\left(t^{i}\right)=\sum_{i=i_{0}}^{\infty} x_{i} t^{i} a=x a,
$$


whence $\phi=\mu_{a}$. Thus $\phi$ is determined by $a=\varepsilon(\phi)$ and thus $\varepsilon$ is injective. Since $\varepsilon^{-1}: a \mapsto \mu_{a}$ is a group homomorphism, $\varepsilon$ is an isomorphism.

Lemma 8.3 Let $s \in\{0,1\}^{\mathbb{N}}$ and $x, y \in A$. Let $n_{0} \in \mathbb{N}$ such that $s_{n}=0$ for all $n \in\left\{1,2, \ldots, n_{0}-1\right\}$. Then

$$
\left|\eta_{s}(x, y)\right| \leq p^{-n_{0}}|x|
$$

Notably, $\left|\eta_{s}(x, y)\right| \leq p^{-1}|x|<|x|$ if $x \neq 0$.

Proof. For all $x, y \in A$, we have

$$
\eta_{s}(x, y)=\sum_{n=n_{0}}^{\infty} s_{n} t^{n} \omega_{2 n}(x, y),
$$

where $\left|s_{n} t^{n} \omega_{2 n}(x, y)\right| \leq p^{-n}\left|\omega_{2 n}(x, y)\right| \leq p^{-n}|x| \leq p^{-n_{0}}|x|$ for all $n \geq n_{0}$, using (44) for the second inequality. We deduce that each finite partial sum in (47) has absolute value $\leq p^{-n_{0}}|x|$, whence so does the limit $\eta_{s}(x, y)$.

Lemma 8.4 For each $s \in\{0,1\}^{\mathbb{N}}$ with $s \neq 0$, the centre of $A \times_{\eta_{s}} A$ is $A \times\{0\}$. If $s=0$, then $A \times{ }_{\eta_{s}} A=A \times A$ is abelian.

Proof. The last assertion being trivial, let us assume that $s \neq 0$ now. Let $x=\left(a_{1}, b_{1}\right) \in A \times_{\eta_{s}} A$. Then $x y=\left(a_{1}+a_{2}+\eta_{s}\left(b_{1}, b_{2}\right), b_{1}+b_{2}\right)$ and $y x=\left(a_{1}+a_{2}+\eta_{s}\left(b_{2}, b_{1}\right), b_{1}+b_{2}\right)$ for all $\left(a_{2}, b_{2}\right) \in A \times_{\eta_{s}} A$, whence $x$ is in the centre of $A \times{ }_{\eta_{s}} A$ if and only if

$$
\eta_{s}\left(b_{1}, b_{2}\right)=\eta_{s}\left(b_{2}, b_{1}\right) \text { for all } b_{2} \in A .
$$

This condition is satisfied if $b_{1}=0$. We show that, conversely, (48) implies $b_{1}=0$, from which the assertion follows. In fact, if $b_{1} \neq 0$, then $b_{1}=$ $\sum_{i=i_{0}}^{\infty} x_{i} t^{i}$ for some $i_{0} \in \mathbb{Z}$ and elements $x_{i} \in \mathbb{F}_{p}$ with $x_{i_{0}} \neq 0$. Let $n_{0} \in \mathbb{N}$ be minimal subject to $s_{n_{0}} \neq 0$. Then

$$
\eta_{s}\left(b_{1}, t^{2 n_{0}+i_{0}}\right)=s_{n_{0}} t^{n_{0}} x_{i_{0}} t^{i_{0}}
$$

with $\left|\eta_{s}\left(b_{1}, t^{2 n_{0}+i_{0}}\right)\right|=p^{-n_{0}-i_{0}}$ but $\left|\eta_{s}\left(t^{2 n_{0}+i_{0}}, b_{1}\right)\right|<\left|t^{2 n_{0}+i_{0}}\right|=p^{-2 n_{0}-i_{0}}<$ $p^{-n_{0}-i_{0}}$, by Lemma 8.3. Thus $\eta_{s}\left(t^{2 n_{0}+i_{0}}, b_{1}\right) \neq \eta_{s}\left(b_{1}, t^{2 n_{0}+i_{0}}\right)$.

Proof of Theorem 8.1. Let $\psi: A \times_{\eta_{s}} A \rightarrow A \times_{\eta_{r}} A$ be as described in the theorem. Lemma 8.4 implies that $r=0$ if and only if $s=0$. We may 
therefore assume now that both $r$ and $s$ are non-zero.

The isomorphism $\psi$ maps the centre of $A \times_{\eta_{s}} A$ onto the centre of $A \times_{\eta_{r}} A$. Using Lemma 8.4, we deduce that

$$
\psi(A \times\{0\})=A \times\{0\} .
$$

In view of (49), we can apply Lemma 6.2, and deduce that there are $\sigma, \tau \in$ $\operatorname{Aut}(A, \alpha)$ and $\beta \in B_{\text {eq }}^{2}(A, A)$ such that

$$
\eta_{r}=\sigma \circ \eta_{s} \circ(\tau \times \tau)+\beta .
$$

By Lemma 8.2, there exist $a, b \in \mathbb{F}_{p}((t))^{\times}$such that $\sigma(x)=a x$ and $\tau(x)=b x$ for all $x \in A$. Thus

$$
\eta_{r}(x, y)=a \eta_{s}(b x, b y)+\beta(x, y) \text { for all } x, y \in A .
$$

Let $k \in \mathbb{Z}$ such that $\left|t^{k} b\right|=1$. Since $\eta_{s}(b x, b y)=t^{-k} \eta_{s}\left(t^{k} b x, t^{k} b y\right)$ for all $x, y \in A$ by equivariance, after replacing $b$ with $t^{k} b$ and $a$ with $t^{-k} a$ we may assume that $|b|=1$. There exists a continuous function $f: A \rightarrow A$ with $f(0)=0$ such that

$$
\beta(x, y)=f(x)+f(y)-f(x+y) \text { for all } x, y \in A .
$$

Then $\beta$ is symmetric:

$$
\beta(x, y)=\beta(y, x) \quad \text { for all } x, y \in A \text {. }
$$

Hence, we have

$$
\eta_{r}(x, y)-\eta_{r}(y, x)=a \eta_{s}(b x, b y)-a \eta_{s}(b y, b x) \text { for all } x, y \in A \text {. }
$$

Let $n_{0} \in \mathbb{N}$ be minimal such that $r_{n_{0}} \neq 0$; let $m_{0} \in \mathbb{N}$ be minimal with $s_{m_{0}} \neq 0$. We claim that

$$
\left|\eta_{r}(x, y)-\eta_{r}(y, x)\right|=p^{-n_{0}}
$$

for all $x, y \in A$ such that $|x|=1$ and $|y|=p^{-2 n_{0}}$. If this is true, then the left-hand side of the identity

$$
\eta_{r}\left(b^{-1} t^{0}, b^{-1} t^{2 n_{0}}\right)-\eta_{r}\left(b^{-1} t^{2 n_{0}}, b^{-1} t^{0}\right)=a \eta_{s}\left(t^{0}, t^{2 n_{0}}\right)-\underbrace{a \eta_{s}\left(t^{2 n_{0}}, t^{0}\right)}_{=0}=a t^{n_{0}} s_{n_{0}}
$$


(which follows from (50) and (43)) has absolute value $p^{-n_{0}}$, whence it is nonzero. Hence also the right-hand side is non-zero and thus $s_{n_{0}} \neq 0$, whence $m_{0} \leq n_{0}$. Hence $s_{n_{0}}=1$, whence the right-hand side of (52) has absolute value $|a| p^{-n_{0}}$. As the left-hand side has absolute value $p^{-n_{0}}$, we deduce that

$$
|a|=1
$$

Reversing the roles of $r$ and $s$, we see that also $n_{0} \leq m_{0}$ and thus $n_{0}=m_{0}$.

To establish the claim (151), write $x=\sum_{i=0}^{\infty} x_{i} t^{i}$ and $y=\sum_{j=2 n_{0}}^{\infty} y_{j} t^{j}$ with $x_{i}, y_{j} \in \mathbb{F}_{p}$ and $x_{0} \neq 0$ as well as $y_{2 n_{0}} \neq 0$. Then

$$
\eta_{r}(x, y)=\eta_{r}\left(x_{0} t^{0}, y_{2 n_{0}} t^{2 n_{0}}\right)+\eta_{r}\left(x_{0} t^{0}, y^{\prime}\right)+\eta_{r}\left(x^{\prime}, y\right)
$$

with $x^{\prime}:=\sum_{i=1}^{\infty} x_{i} t^{i}$ and $y^{\prime}:=\sum_{j=2 n_{0}+1}^{\infty} y_{j} t^{j}$. The first summand in (54) is $t^{n_{0}} r_{n_{0}} x_{0} y_{2 n_{0}}=t^{n_{0}} x_{0} y_{2 n_{0}}$, which has absolute value $p^{-n_{0}}$. Since $\omega_{2 n_{0}}\left(x_{0} t^{0}, y^{\prime}\right)=$ 0 , the second summand in (54) is

$$
\sum_{n=n_{0}+1}^{\infty} r_{n} t^{n} \omega_{2 n}\left(x_{0} t^{0}, y^{\prime}\right)
$$

by Lemma 8.3, its absolute value is $\leq p^{-n_{0}-1}\left|x_{0} t^{0}\right|=p^{-n_{0}-1}<p^{-n_{0}}$. As $\left|x^{\prime}\right| \leq p^{-1}$, Lemma 8.3 yields $\left|\eta_{r}\left(x^{\prime}, y\right)\right| \leq p^{-n_{0}}\left|x^{\prime}\right| \leq p^{-n_{0}-1}<p^{-n_{0}}$ for the third summand in (54). Using (3), we deduce that

$$
\left|\eta_{r}(x, y)\right|=p^{-n_{0}}
$$

Now $\left|\eta_{r}(y, x)\right| \leq p^{-n_{0}}|y|=p^{-n_{0}} p^{-2 n_{0}}<p^{-n_{0}}$, by Lemma 8.3. Thus, using (3), we find that $\left|\eta_{r}(x, y)-\eta_{r}(y, x)\right|=\left|\eta_{r}(x, y)\right|=p^{-n_{0}}$. The claim is established.

We now show that $r_{m}=s_{m}$ for all $m>n_{0}$ (which completes the proof). Given $m$, we claim that, for all $x, y \in A$ with $|x|=1$ and $|y|=p^{-2 m}$, the absolute value $\left|\eta_{r}(x, y)-\eta_{r}(y, x)\right|$ equals $p^{-m}$ if $r_{m} \neq 0$, but is $<p^{-m}$ if $r_{m}=0$. If this is true, then the left-hand side of the identity

$$
\left|\eta_{r}\left(b^{-1} t^{0}, b^{-1} t^{2 m}\right)-\eta_{r}\left(b^{-1} t^{2 m}, b^{-1} t^{0}\right)\right|=\left|a \eta_{s}\left(t^{0}, t^{2 m}\right)\right|=p^{-m}\left|s_{m}\right|
$$

has absolute value $p^{-m}$ if and only if $r_{m} \neq 0$. As the right-hand side has absolute value $p^{-m}$ if and only if $s_{m} \neq 0$, we deduce that $r_{m}=s_{m}$.

To establish the claim, write $x=\sum_{i=0}^{\infty} x_{i} t^{i}$ and $y=\sum_{j=2 m}^{\infty} y_{j} t^{j}$ with $x_{i}, y_{j} \in$ 
$\mathbb{F}_{p}$ and $x_{0} \neq 0$ as well as $y_{2 m} \neq 0$. For $j \in \mathbb{N}$ such that $j<2 m$, set $y_{j}:=0$. Then

$$
\eta_{r}(x, y)=\eta_{r}\left(x_{0} t^{0}, y_{2 m} t^{2 m}\right)+\eta_{r}\left(x_{0} t^{0}, y^{\prime}\right)+\eta_{r}\left(x^{\prime}, y\right)
$$

with $x^{\prime}:=\sum_{i=1}^{\infty} x_{i} t^{i}$ and $y^{\prime}:=\sum_{j=2 m+1}^{\infty} y_{j} t^{j}$. The first summand in (56) is $t^{m} r_{m} x_{0} y_{2 m}$; its absolute value is $p^{-m}$ if $r_{m} \neq 0$ and vanishes if $r_{m}=0$. Since $\omega_{2 n}\left(x_{0} t^{0}, y^{\prime}\right)=0$ for $n \in\{1, \ldots, m\}$, the second summand is

$$
\sum_{n=m+1}^{\infty} r_{n} t^{n} \omega_{2 n}\left(x_{0} t^{0}, y^{\prime}\right)=\sum_{n=m+1}^{\infty} r_{n} t^{n} x_{0} y_{2 n}
$$

whence it has absolute value $\leq p^{-m-1}<p^{-m}$. Since $y_{i+2 n} \neq 0$ implies $i+2 n \geq 2 m$ and thus $i \geq 2(m-n)$, the third summand is

$$
\sum_{n=n_{0}}^{\infty} \sum_{i=1}^{\infty} r_{n} t^{n+i} x_{i} y_{i+2 n}=\sum_{n=n_{0}}^{\infty} \sum_{i=\max \{1,2(m-n)\}}^{\infty} r_{n} t^{n+i} x_{i} y_{i+2 n}
$$

For $n \in\left\{n_{0}, \ldots, m-1\right\}$, we have $2(m-n) \geq 2$ and thus $\max \{1,2(m-n)\}=$ $2(m-n)$, entailing that $n+i \geq n+2(m-n)=2 m-n \geq 2 m-(m-1)=m+1$ and thus $\left|t^{n+i}\right| \leq p^{-m-1}$ on the right hand side of (57). For $n \geq m$, we have $2(m-n) \leq 0$ and thus $\max \{1,2(m-n)\}=1$, whence $n+i \geq n+1 \geq m+1$ and hence $\left|t^{n+i}\right| \leq p^{-m-1}$. As a consequence, the third summand in (156) has absolute value $\leq p^{-m-1}$. Applying (3) to the summands in (56), we see that

$$
\left|\eta_{r}(x, y)\right|=p^{-m} \text { if } r_{m} \neq 0 \text {, while }\left|\eta_{r}(x, y)\right| \leq p^{-m-1}<p^{-m} \text { if } r_{m}=0,
$$

by the ultrametric inequality. By Lemma 8.3, we have

$$
\left|\eta_{r}(y, x)\right|<|y|=p^{-2 m}<p^{-m} .
$$

Using (13) and the ultrametric inequality, the claim follows from (158) and (159).

\section{Open Problems}

If $p$ is a prime and $(G, \alpha)$ is a torsion contraction group all of whose composition factors are isomorphic to $\mathbb{F}_{p}((t))$, then $G$ is soluble (as all composition factors are abelian) and $G$ has a compact open subgroup which is a pro- $p$ group and hence pro-nilpotent. The following question is natural: 
Question 1. Let $p$ be a prime and $(G, \alpha)$ be a torsion contraction group all of whose composition factors are isomorphic to $\mathbb{F}_{p}((t))$. Will $G$ be nilpotent?

The answer is positive if $G$ is an analytic Lie group over a local field of positive characteristic and $\alpha$ an analytic automorphism [6], but the general case is open. The case of two composition factors should be tackled first 5

Question 2. Let $p$ be a prime and $(\widehat{G}, \alpha)$ be a torsion contraction group which is an extension $\{e\} \rightarrow \mathbb{F}_{p}((t)) \rightarrow \widehat{G} \rightarrow \mathbb{F}_{p}((t)) \rightarrow\{e\}$. Will $\widehat{G}$ be nilpotent?

We mention some immediate observations.

Proposition 9.1 The following conditions are equivalent.

(a) There exists an extension $\{e\} \rightarrow \mathbb{F}_{p}((t)) \rightarrow \widehat{G} \rightarrow \mathbb{F}_{p}((t)) \rightarrow\{e\}$ of contraction groups such that $\widehat{G}$ is not nilpotent.

(b) There exists an extension $\{e\} \rightarrow \mathbb{F}_{p}((t)) \stackrel{\iota}{\rightarrow} \widehat{G} \rightarrow \mathbb{F}_{p}((t)) \rightarrow\{e\}$ of contraction groups such that $\widehat{G}$ has trivial centre, $Z(\widehat{G})=\{e\}$.

(c) There exists a continuous homomorphism

$$
\gamma:\left(\mathbb{F}_{p}((t)),+\right) \rightarrow \operatorname{Aut}\left(\mathbb{F}_{p}((t)),+\right)
$$

to the topological group of all automorphisms of the locally compact group $\left(\mathbb{F}_{p}((t)),+\right)$ such that

$$
\gamma(t x)(t y)=t \gamma(x)(y) \text { for all } x, y \in \mathbb{F}_{p}((t))
$$

and $\gamma(z) \neq$ id for some $z \in \mathbb{F}_{p}((t))$.

Proof. Since every non-trivial nilpotent group has non-trivial centre, (b) implies (a). By Lemma 6.1, (a) implies (b). Next, (b) implies (c) since $\widehat{G}$ as in (b) has $\iota\left(\mathbb{F}_{p}((t))\right) \nsubseteq Z Z(\widehat{G})$ and hence induces a non-trivial continuous homomorphism $\gamma: \mathbb{F}_{p}((t)) \rightarrow \operatorname{Aut}\left(\mathbb{F}_{p}((t))\right)$ as described in Example A.5 (which satisfies (60) due to (66) ).

$(\mathrm{c}) \Rightarrow(\mathrm{b})$ : Let $\gamma$ be as in (c). Then $\mathbb{F}_{p}((t)) \times\{0\}$ is not contained in the centre of the semi-direct product $\mathbb{F}_{p}((t)) \rtimes_{\gamma} \mathbb{F}_{p}((t))$, whence the latter has trivial centre (by Lemma 6.1) 6

\footnotetext{
${ }^{5}$ The authors are grateful to P.-E. Caprace for prompting a similar question.

${ }^{6}$ Note that the semi-direct product is a contraction group with contractive automorphism $(x, y) \mapsto(t x, t y)$.
} 
Remark 9.2 If $\gamma: \mathbb{F}_{p}((t)) \rightarrow \operatorname{Aut}\left(\mathbb{F}_{p}((t))\right)$ is a continuous homomorphism such that (60) holds, define $\theta:=\gamma\left(t^{0}\right)$. Then

$$
\gamma\left(\sum_{n=N}^{\infty} a_{n} t^{n}\right)=\lim _{n \rightarrow \infty} \gamma\left(t^{n}\right)^{a_{n}} \circ \cdots \circ \gamma\left(t^{N}\right)^{a_{N}}
$$

$\operatorname{in} \operatorname{Aut}\left(\mathbb{F}_{p}((t))\right)$, for all $N \in \mathbb{Z}$ and $a_{N}, a_{N+1}, \ldots \in \mathbb{F}_{p}$. Moreover,

$$
\gamma\left(t^{n}\right)(x)=t^{n} \theta\left(t^{-n} x\right) \text { for all } n \in \mathbb{Z} \text { and } x \in \mathbb{F}_{p}((t))
$$

Thus $\theta=\mathrm{id}$ implies $\gamma(x)=\mathrm{id}$ for all $x \in \mathbb{F}_{p}((t))$. Since $\gamma$ is a homomorphism and $\mathbb{F}_{p}((t))$ is abelian, we have $\gamma\left(t^{n}\right) \circ \gamma\left(t^{m}\right)=\gamma\left(t^{m}\right) \circ \gamma\left(t^{n}\right)$ for all $n, m \in \mathbb{Z}$. Moreover, $\gamma(x)^{p}=\gamma(p x)=\gamma(0)=$ id for all $x \in \mathbb{F}_{p}((t))$.

Remark 9.3 If $\theta \neq$ id in Remark 9.2, then $\theta$ cannot be of the most naïve form, i.e., it cannot come from a permutation $\pi$ of $\mathbb{Z}$ in the sense that

$$
\theta\left(t^{k}\right)=t^{\pi(k)} \text { for all } k \in \mathbb{Z}
$$

In fact, since $\theta \neq$ id and the subgroup generated by $\left\{t^{k}: k \in \mathbb{Z}\right\}$ is dense in $\mathbb{F}_{p}((t))$, we would have $\ell:=\pi(k) \neq k$ for some $k \in \mathbb{Z}$. Since $\pi^{p}=\mathrm{id}$, after replacing $k$ by another element in its $\langle\pi\rangle$-orbit if necessary, we may assume that $\ell>k$. Set $\Delta:=\ell-k>0$. Then

$$
\gamma\left(t^{n \Delta}\right)\left(t^{n \Delta+k}\right)=\gamma\left(t^{n \Delta}\right)\left(t^{n \Delta} t^{k}\right)=t^{n \Delta} \theta\left(t^{k}\right)=t^{(n+1) \Delta+k} \text { for all } n \in \mathbb{N}_{0},
$$

using (62). Thus

$$
\left(\gamma\left(t^{n \Delta}\right) \circ \cdots \circ \gamma\left(t^{\Delta}\right) \circ \gamma\left(t^{0}\right)\right)\left(t^{k}\right)=t^{(n+1) \Delta+k} .
$$

For $x:=\sum_{n=0}^{\infty} t^{n \Delta} \in \mathbb{F}_{p}((t))$, we then have

$$
\gamma(x)\left(t^{k}\right)=\lim _{n \rightarrow \infty}\left(\gamma\left(t^{n \Delta}\right) \circ \cdots \circ \gamma\left(t^{\Delta}\right) \circ \gamma\left(t^{0}\right)\right)\left(t^{k}\right)=\lim _{n \rightarrow \infty} t^{(n+1) \Delta+k}=0 .
$$

But $\gamma(x)\left(t^{k}\right) \neq 0$ as $t^{k} \neq 0$ and $\gamma(x)$ is an automorphism, contradiction.

Remark 9.4 Every homomorphism $\gamma$ as in Proposition 9.1(c) satisfies

$$
\left(\forall 0 \neq y \in \mathbb{F}_{p}((t))\right)(\exists k \in \mathbb{Z}) \gamma\left(t^{k}\right)(y) \neq y .
$$


In fact, if there were $0 \neq y \in \mathbb{F}_{p}((t))$ such that $\gamma\left(t^{k}\right)(y)=y$ for all $k \in \mathbb{Z}$, then we would have $\gamma(x)(y)=y$ for all $x \in \mathbb{F}_{p}((t))$, by (61). Thus $(y, 0)$ would be in the centre of the semidirect product $\widehat{G}:=\mathbb{F}_{p}((t)) \rtimes_{\gamma} \mathbb{F}_{p}((t))$, since

$$
(a, x)(y, 0)(a, x)^{-1}=(\gamma(x)(y), 0)=(y, 0) \text { for all }(a, x) \in \widehat{G} .
$$

As a consequence, $\mathbb{F}_{p}((t)) \times\{0\}$ would be in the centre of $\widehat{G}$, by Lemma 6.1 , entailing that $\gamma(x)=\mathrm{id}$ for each $x \in \mathbb{F}_{p}((t))$. We have reached a contradiction.

Remark 9.5 Specializing to $p=2$, consider a continuous homomorphism $\gamma: \mathbb{F}_{2}((t)) \rightarrow \operatorname{Aut}\left(\mathbb{F}_{2}((t))\right)$ such that $(\underline{60})$ holds. Then we find a non-zero joint fixed vector $z$ for $\gamma\left(t^{k_{1}}\right), \ldots, \gamma\left(t^{k_{n}}\right)$ in the $\mathbb{F}_{2}$-vector space $\mathbb{F}_{2}((t))$ for each finite subset $\left\{k_{1}, \ldots, k_{n}\right\} \subseteq \mathbb{Z}$, as we presently verify. However, it is not clear whether we can find a non-zero joint fixed vector $y$ for all $\gamma\left(t^{k}\right)$ with $k \in \mathbb{Z}$ simultaneously. If $y$ always exists, then $\gamma(x)=\mathrm{id}$ for all $x \in \mathbb{F}_{2}((t)$ ) (by the argument in Remark 9.4), whence every extension $\widehat{G}$ of $\mathbb{F}_{2}((t))$ by itself would be nilpotent, by Proposition 9.1 .

For the verification, we use that $\gamma\left(t^{k_{j}}\right)^{2}=$ id for $j \in\{1, \ldots, n\}$. Start with a vector $0 \neq y_{0} \in \mathbb{F}_{2}((t))$. We define non-zero elements $y_{1}, \ldots, y_{n} \in \mathbb{F}_{2}((t))$ such that $y_{j}$ is a fixed vector for all of $\gamma\left(t^{k_{1}}\right), \ldots, \gamma\left(t^{k_{j}}\right)$, as follows: Recursively, for $j \in\{1, \ldots, n\}$, we take $y_{j}:=y_{j-1}$ if $\gamma\left(t^{k_{j}}\right)\left(y_{j-1}\right)=y_{j-1}$ and $y_{j}:=$ $y_{j-1}+\gamma\left(t^{k_{j}}\right)\left(y_{j-1}\right)$ otherwise. Then $y_{j}$ is a fixed vector for $\gamma\left(t^{k_{j}}\right)$ and also for $\gamma\left(t^{j_{1}}\right), \ldots, \gamma\left(t^{j-1}\right)$, as $y_{j-1}$ was a fixed vector for these and $\gamma\left(t^{k_{j}}\right)$ leaves the 1-eigenspace of $\gamma\left(t^{k_{i}}\right)$ invariant for all $i \in\{1, \ldots, j-1\}$ since $\gamma\left(t^{k_{i}}\right)$ and $\gamma\left(t^{k_{j}}\right)$ commute. Now set $z:=y_{n}$.

Nilpotency of contraction groups can be checked on open subgroups.

Proposition 9.6 Let $\alpha: G \rightarrow G$ be a contractive automorphism of a topological group $G$. Then the following holds:

(a) If $G$ has a nilpotent open subgroup, then $G$ is nilpotent.

(b) If $G$ has a soluble open subgroup, then $G$ is soluble.

Our proof uses a well-known fact (whose simple proof is left to the reader):

Lemma 9.7 Let $\mathcal{A}$ and $\mathcal{B}$ be sets of subgroups of a group $G$ which are directed upward under inclusion. Then $\mathcal{C}:=\{[A, B]:(A, B) \in \mathcal{A} \times \mathcal{B}\}$ is directed under inclusion, the unions $\cup \mathcal{A}:=\cup_{A \in \mathcal{A}} A, \cup \mathcal{B}$, and $\cup \mathcal{C}$ are subgroups of $G$, and $[\cup \mathcal{A}, \cup \mathcal{B}]=\cup \mathcal{C}$. 
Proof of Proposition 9.6. Let $U$ be an open subgroup of $G$ which is nilpotent (resp., soluble). Let $n \in \mathbb{N}$ such that $C^{n}(U)=\{e\}$ (resp., $U^{(n)}=$ $\{e\})$. Let $\Phi \subseteq G$ be a finite subset and $H:=\langle\Phi\rangle$. Since $\alpha$ is contractive, there is $m \in \overline{\mathbb{N}}$ such that $\alpha^{m}(\Phi) \subseteq U$ and thus also $\alpha^{m}(H) \subseteq U$. Then

$$
\alpha^{m}\left(C^{n}(H)\right) \subseteq C^{n}(U) \text { and } \quad \alpha^{m}\left(H^{(n)}\right) \subseteq U^{(n)},
$$

showing that $C^{n}(H)=\{e\}$ (resp., $H^{(n)}=\{e\}$ ). Since $G$ is the union of its directed set $\mathcal{F}$ of finitely generated subgroups, Lemma 9.7 implies that

$$
C^{n}(G)=\bigcup_{F \in \mathcal{F}} C^{n}(F)=\{e\}, \quad \text { resp. }, \quad G^{(n)}=\bigcup_{F \in \mathcal{F}} F^{(n)}=\{e\} .
$$

Thus $G$ is nilpotent (resp., soluble).

\section{A Extensions of contraction groups with abelian kernel, and cohomology}

Given a group $G$ and a homomorphism $\gamma: G \rightarrow \operatorname{Aut}(A)$ to the automorphism group of an abelian group $A$, it is well-known that the cohomology classes $[\omega] \in H^{2}(G, A)$ of 2-cocycles $\omega: G \times G \rightarrow A$ parametrize the equivalence classes of those group extensions

$$
\{e\} \rightarrow A \stackrel{\iota}{\rightarrow} \widehat{G} \stackrel{q}{\rightarrow} G \rightarrow\{e\}
$$

which induce $\gamma$ in the sense that

$$
\iota(\gamma(q(x))(a))=x \iota(a) x^{-1} \text { for all } a \in A \text { and } x \in \widehat{G} .
$$

In this appendix, we give an analogous treatment for extensions of contraction groups with abelian kernel. We focus on the specific aspects of this situation and omit calculations which are analogous to the classical case of group extensions, as treated in many books (like [3, Chapter IV.3]; cf. [11] and [12]).

A.1 Throughout the appendix, $\left(A, \alpha_{A}\right)$ and $(G, \alpha)$ are locally compact contraction groups, such that $A$ is abelian. We fix a continuous homomorphism

$$
\gamma: G \rightarrow \operatorname{Aut}(A)
$$


to the group of automorphisms of the locally compact group $A$, such that

$$
\gamma(\alpha(g))=\alpha_{A} \circ \gamma(g) \circ \alpha_{A}^{-1} \text { for all } g \in G \text {. }
$$

Then $G \times A \rightarrow A,(g, a) \mapsto g \cdot a:=\gamma(g)(a)$ is a continuous action of $G$ on $A$ and (65) is equivalent to the condition

$$
\alpha(g) \cdot \alpha_{A}(a)=\alpha_{A}(g . a) \quad \text { for all } a \in A \text { and } g \in G .
$$

The binary group multiplication on $A$ will be written additively, while multiplicative notation is used for $G$. The neutral elements are $0 \in A$ and $e \in G$.

Definition A.2 A continuous mapping $\omega: G \times G \rightarrow A$ is called a 2-cocycle of contraction groups if it is continuous, satisfies the cocycle condition

$$
g_{1} \cdot \omega\left(g_{2}, g_{3}\right)-\omega\left(g_{1} g_{2}, g_{3}\right)+\omega\left(g_{1}, g_{2} g_{3}\right)-\omega\left(g_{1}, g_{2}\right)=0 \text { for all } g_{1}, g_{2}, g_{3} \in G
$$

and is equivariant in the sense that

$$
\omega\left(\alpha\left(g_{1}\right), \alpha\left(g_{2}\right)\right)=\alpha_{A}\left(\omega\left(g_{1}, g_{2}\right)\right) \quad \text { for all } g_{1}, g_{2} \in G .
$$

The set $Z_{\text {eq }}^{2}(G, A)$ of all such $\omega$ is a subgroup of the abelian group $A^{G \times G}$.

A.3 By the equivariance condition, $\omega(e, e)=\omega\left(\alpha^{n}(e), \alpha^{n}(e)\right)=\alpha_{A}^{n}(\omega(e, e))$ for all $n \in \mathbb{N}$. As $\alpha_{A}$ is contractive, letting $n \rightarrow \infty$ we find that

$$
\omega(e, e)=0
$$

i.e., the cocycles we consider are always normalized.

A.4 Applying the cocycle condition (67) to $(e, e, g),(g, e, e)$ and $\left(g^{-1}, g, g^{-1}\right)$, respectively, in place of $\left(g_{1}, g_{2}, g_{3}\right)$, we deduce that

$$
\begin{aligned}
\omega(e, g) & =\omega(e, e)=0, \\
\omega(g, e) & =g \cdot \omega(e, e)=0 \text { and } \\
\omega\left(g^{-1}, g\right) & =g^{-1} \cdot \omega\left(g, g^{-1}\right) \text { for all } g \in G ;
\end{aligned}
$$

these identities are useful for the omitted standard calculations. 
Example A.5 Consider an extension $\{e\} \rightarrow A \stackrel{\iota}{\rightarrow} \widehat{G} \stackrel{q}{\rightarrow} G \rightarrow\{e\}$ of contraction groups and let $\widehat{\alpha}$ be the contractive automorphism of $\widehat{G}$. Let $\sigma: G \rightarrow \widehat{G}$ be an equivariant continuous section to $q$, as provided by Theorem $\mathrm{C}$. Then

$$
G \times A \rightarrow A, \quad \text { g.a }:=\iota^{-1}\left(\sigma(g) \iota(a) \sigma(g)^{-1}\right)
$$

is a continuous left action of $G$ on $A$ such that $\gamma(g): A \rightarrow A, \gamma(g)(a):=$ $g . a$ is an automorphism of $A$ for each $g \in G$. By [1.5, the corresponding homomorphism

$$
\gamma: G \rightarrow \operatorname{Aut}(A), \quad g \mapsto \gamma(g)
$$

is continuous, and (66) holds by construction. A standard calculation shows that the map

$$
\omega: G \times G \rightarrow A, \quad\left(g_{1}, g_{2}\right) \mapsto \iota^{-1}\left(\sigma\left(g_{1}\right) \sigma\left(g_{2}\right) \sigma\left(g_{1} g_{2}\right)^{-1}\right)
$$

satisfies the cocycle condition (67); as $\omega$ is continuous and inherits (68) from the equivariance of $\sigma$, we deduce that $\omega \in Z_{\text {eq }}^{2}(G, A)$. The map

$$
\pi: A \times G \rightarrow \widehat{G}, \quad(a, g) \mapsto \iota(a) \sigma(g)
$$

is a bijection. The unique group multiplication on $A \times G$ making $\pi$ an isomorphism of groups is given by

$$
\left(a_{1}, g_{1}\right)\left(a_{2}, g_{2}\right):=\left(a_{1}+g_{1} \cdot a_{2}+\omega\left(g_{1}, g_{2}\right), g_{1} g_{2}\right)
$$

for $\left(a_{1}, g_{1}\right),\left(a_{2}, g_{2}\right) \in A \times G$, as

$$
\begin{aligned}
\pi\left(a_{1}, g_{1}\right) \pi\left(a_{2}, g_{2}\right) & =\iota\left(a_{1}\right) \sigma\left(g_{1}\right) \iota\left(a_{2}\right) \sigma\left(g_{2}\right) \\
& =\iota\left(a_{1}\right) \sigma\left(g_{1}\right) \iota\left(a_{2}\right) \sigma\left(g_{1}\right)^{-1} \sigma\left(g_{1}\right) \sigma\left(g_{2}\right) \sigma\left(g_{1} g_{2}\right)^{-1} \sigma\left(g_{1} g_{2}\right) \\
& =\pi\left(a_{1}+g_{1} \cdot a_{2}+\omega\left(g_{1}, g_{2}\right), g_{1} g_{2}\right) .
\end{aligned}
$$

Since $\pi(0, e)=e$, the neutral element is $\pi^{-1}(e)=(0, e)$. As the product topology on $A \times G$ makes $\pi$ a homeomorphism, it makes $A \times G$ a topological group, which we denote by $A \times_{\omega} G$. The map $\alpha_{A} \times \alpha$ is a contractive automorphism of $A \times G$, since $\pi \circ\left(\alpha_{A} \times \alpha\right)=\widehat{\alpha} \circ \pi$.

Example A.5 motivates the following proposition, in the setting of A.1. 
Proposition A.6 Given $\omega \in Z_{\mathrm{eq}}^{2}(G, A)$, the binary operation described in (74) makes $A \times G$ a locally compact contraction group $A \times{ }_{\omega} G$ when endowed with the product topology and the contractive automorphism $\alpha_{A} \times \alpha$. The mappings $\iota: A \rightarrow A \times{ }_{\omega} G, a \mapsto(a, e)$ and $\operatorname{pr}_{2}: A \times_{\omega} G \rightarrow G,(a, g) \mapsto g$ are morphisms of contraction groups and

$$
\{e\} \rightarrow A \stackrel{\iota}{\rightarrow} A \times_{\omega} G \stackrel{\mathrm{pr}_{2}}{\rightarrow} G \rightarrow\{e\}
$$

is an extension of contraction groups which induces $\gamma$. The mapping $\sigma: G \rightarrow A \times_{\omega} G, g \mapsto(0, g)$ is an equivariant continuous section for $\mathrm{pr}_{2}$ such that

$$
\omega\left(g_{1}, g_{2}\right)=\sigma\left(g_{1}\right) \sigma\left(g_{2}\right) \sigma\left(g_{1} g_{2}\right)^{-1} \text { for all } g_{1}, g_{2} \in G
$$

and $\iota(\gamma(g)(a))=\sigma(g) \iota(a) \sigma(g)^{-1}$ for all $g \in G$ and $a \in A$.

Proof. Classical calculations show that the binary operation (74) makes $A \times_{\omega} G$ a group with neutral element $(0, e)$, that $\iota$ and $\mathrm{pr}_{2}$ are group homomorphisms, (76) and the final identity hold, and that (75) is an exact sequence of groups which induces $\gamma$. Inverses are given by

$$
(a, g)^{-1}=\left(-g^{-1} \cdot a-g^{-1} \cdot \omega\left(g, g^{-1}\right), g^{-1}\right) \text { for }(a, g) \in A \times_{\omega} G .
$$

Since $A$ and $G$ are topological groups and both $\omega$ and the left $G$-action on $A$ are continuous, we deduce from the explicit formulas (174) and (77) that $A \times{ }_{\omega} G$ is a topological group. Using (66) , one finds that the homeomorphism $\alpha_{A} \times \alpha$ is a homomorphism of groups and hence a contractive automorphism. To complete the proof, it suffices to observe that $\operatorname{pr}_{2} \circ\left(\alpha_{A} \times \alpha\right)=\alpha \circ \operatorname{pr}_{2}$ and $\left(\alpha_{A} \times \alpha\right) \circ \iota=\iota \circ \alpha_{A}$.

Definition A.7 A 2-coboundary of contraction groups is a map of the form

$$
\omega_{f}: G \times G \rightarrow A, \quad\left(g_{1}, g_{2}\right) \mapsto g_{1} \cdot f\left(g_{2}\right)-f\left(g_{1} g_{2}\right)+f\left(g_{1}\right),
$$

where $f: G \rightarrow A$ is a continuous map which is equivariant in the sense that

$$
f \circ \alpha=\alpha_{A} \circ f \text {. }
$$

A standard calculation shows that $\omega_{f}$ satisfies the cocycle condition. As it is continuous and inherits the equivariance property (68) from (78), we deduce that $\omega_{f}$ is a 2-cocycle of contraction groups. Thus, the set $B_{\text {eq }}^{2}(G, A)$ of all $\omega_{f}$ is a subgroup of the abelian group $Z_{\text {eq }}^{2}(G, A)$. 
Definition A.8 We write $H_{\mathrm{eq}}^{2}(G, A):=Z_{\mathrm{eq}}^{2}(G, A) / B_{\mathrm{eq}}^{2}(G, A)$ and call $[\omega]:=$ $\omega+B_{\mathrm{eq}}^{2}(G, A) \in H_{\mathrm{eq}}^{2}(G, A)$ the cohomology class of $\omega \in Z_{\mathrm{eq}}^{2}(G, A)$.

We say that two extensions $\{e\} \rightarrow A \stackrel{\iota^{\prime}}{\rightarrow} \widehat{G}_{1} \stackrel{q_{1}}{\rightarrow} G \rightarrow\{e\}$ and $\{e\} \rightarrow A \stackrel{\iota}{\rightarrow}$ $\widehat{G}_{2} \stackrel{q_{2}}{\rightarrow} G \rightarrow\{e\}$ of contraction groups inducing $\gamma$ are equivalent if there exists an isomorphism of contraction groups $\psi: \widehat{G}_{1} \rightarrow \widehat{G}_{2}$ such that $\psi \circ \iota_{1}=\iota_{2}$ and $q_{2} \circ \psi=q_{1}$. Given an extension $E:\{e\} \rightarrow A \stackrel{\iota}{\rightarrow} \widehat{G} \stackrel{q}{\rightarrow} G \rightarrow\{e\}$ of contraction groups, let us write $[E]$ for its equivalence class (or simply $[\widehat{G}]$, if $\iota$ and $q$ are understood).

Lemma A.9 If $\omega_{1}, \omega_{2} \in Z_{\mathrm{eq}}^{2}(G, A)$ and $\omega_{2}-\omega_{1} \in B_{\mathrm{eq}}^{2}(G, A)$, then $\left[A \times_{\omega_{1}} G\right]=$ $\left[A \times_{\omega_{2}} G\right]$.

Proof. We have $\omega_{2}-\omega_{1}=\omega_{f}$ for an equivariant continuous map $f: G \rightarrow A$, as in Definition A.7. Now $\psi: A \times_{\omega_{2}} G \rightarrow A \times_{\omega_{1}} G,(a, g) \mapsto(a+f(g), g)$ is a homeomorphism and a homomorphism of groups, by standard calculations. Since $f$ is equivariant, $\psi$ is a morphism of contraction groups.

Lemma A.10 Let $\{e\} \rightarrow A \stackrel{\iota}{\rightarrow} \widehat{G} \stackrel{q}{\rightarrow} G \rightarrow\{e\}$ be an extension of contraction groups inducing $\gamma$. Let $\sigma_{j}: G \rightarrow \widehat{G}$ be an equivariant continuous section for $q$ and $\omega_{j}\left(g_{1}, g_{2}\right):=\iota^{-1}\left(\sigma_{j}\left(g_{1}\right) \sigma_{j}\left(g_{2}\right) \sigma_{j}\left(g_{1} g_{2}\right)^{-1}\right)$ for $j \in\{1,2\}$ and $g_{1}, g_{2} \in G$. Then $\omega_{2}-\omega_{1} \in B_{\text {eq }}^{2}(G, A)$.

Proof. The function $f: G \rightarrow A, f(g):=\iota^{-1}\left(\sigma_{2}(g) \sigma_{1}(g)^{-1}\right)$ is continuous and equivariant. Standard arguments show that $\omega_{2}=\omega_{1}+\omega_{f}$.

Proposition A.11 The assignment $[\omega] \mapsto\left[A \times{ }_{\omega} G\right]$ is a bijection from $H_{\mathrm{eq}}^{2}(G, A)$ onto the set of all equivalence classes of extensions

$$
\{e\} \rightarrow A \rightarrow \widehat{G} \rightarrow G \rightarrow\{e\}
$$

of contraction groups which induce $\gamma$.

Proof. The assignment is well defined by Lemma A.9 and surjective (see Example A.5). To prove injectivity, let $\omega_{1}, \omega_{2} \in Z_{\mathrm{eq}}^{2}(G, A)$ and assume that there exists an isomorphism of contraction groups

$$
\psi: A \times_{\omega_{1}} G \rightarrow A \times_{\omega_{2}} G
$$


such that $\operatorname{pr}_{2} \circ \psi=\operatorname{pr}_{2}$ and $\psi \circ \iota=\iota$ (where $\iota: A \rightarrow A \times G, a \mapsto(a, e)$ ). Then both $\sigma_{1}: G \rightarrow A \times G, g \mapsto(0, g)$ and $\sigma_{2}:=\psi^{-1} \circ \sigma_{1}$ are equivariant sections for $\mathrm{pr}_{2}$, and

$$
\omega_{j}\left(g_{1}, g_{2}\right)=\iota^{-1}\left(\sigma_{j}\left(g_{1}\right) \sigma_{j}\left(g_{2}\right) \sigma_{j}\left(g_{1} g_{2}\right)^{-1}\right)
$$

for $j \in\{1,2\}$ and all $g_{1}, g_{2} \in G$, when the products in (79) are calculated in $A \times_{\omega_{1}} G$. Hence $\omega_{2}-\omega_{1} \in B_{\mathrm{eq}}^{2}(G, A)$, by Lemma A.10.

\section{References}

[1] Baumgartner, U. and G.A. Willis, Contraction groups and scales of automorphisms of totally disconnected locally compact groups, Israel J. Math. 142 (2004), 221-248.

[2] Bourbaki, N., "Lie Groups and Lie Algebras," Chapters 1-3, Springer, Berlin, 1989.

[3] Brown, K. S., "Cohomology of Groups," Graduate Text in Mathematics 87, Springer, New York, 1982.

[4] Dixon, J. D., M. P. F. du Sautoy, A. Mann, and D. Segal, "Analytic Pro- $p$ Groups," Cambridge University Press, Cambridge, ${ }^{2} 1999$.

[5] Fell, J.M. G. and R.S. Doran, "Representations of *-Algebras, Locally Compact Groups, and Banach *-Algebraic Bundles," Academic Press, 1988.

[6] Glöckner, H., Contractible Lie groups over local fields, Math. Z. 260 (2008), 889-904.

[7] Glöckner, H. and G.A. Willis, Classification of the simple factors appearing in composition series of totally disconnected contraction groups, J. Reine Angew. Math. 634 (2010), 141-169.

[8] Hewitt, E. and K. A. Ross, "Abstract Harmonic Analysis I," Springer, New York, ${ }^{2} 1979$.

[9] Jacobson, N., "Basic Algebra I," W. H. Freeman and Company, New York, ${ }^{2} 1985$. 
[10] Lang, S., "Fundamentals of Differential Geometry", Springer, 1999.

[11] Robinson, D. J. S., "A Course in the Theory of Groups," Graduate Text in Mathematics 80, Springer, New York, ${ }^{2} 1995$.

[12] Rotman, J. J., "An Introduction to the Theory of Groups," Springer, New York, ${ }^{4} 1995$.

[13] Schikhof, W.H., "Ultrametric Calculus," Cambridge University Press, 1984.

[14] Serre, J.-P., "Lie Algebras and Lie Groups," Springer, Berlin, ${ }^{2} 1992$.

[15] Siebert, E., Contractive automorphisms on locally compact groups, Math. Z. 191 (1986), 73-90.

[16] Stroppel, M., "Locally Compact Groups," European Mathematical Society Publishing House, Zürich, 2006.

[17] Wang, J.S.P., The Mautner phenomenon for p-adic Lie groups, Math. Z. 185 (1984), 403-412.

[18] Weil, A., "Basic Number Theory," Springer, Berlin, ${ }^{2} 1973$.

[19] Wilson, J.S., "Profinite Groups," Oxford University Press, Oxford, 1998.

Helge Glöckner, Institut für Mathematik, Universität Paderborn, Warburger Str. 100, 33098 Paderborn, Germany, email: glockner@math.upb.de; also conjoint professor at Department of Mathematics, University of Newcastle, Callaghan, NSW 2308, Australia.

George A. Willis, Department of Mathematics, University of Newcastle, Callaghan, NSW 2308, Australia, email: George.Willis@newcastle.edu.au 